\title{
The Hippo Effector Transcriptional Coactivator with PDZ-Binding Motif Cooperates with Oncogenic $\beta$-Catenin to Induce Hepatoblastoma Development in Mice and Humans
}

From the Cancer Center and State Key Laboratory of Biotherapy* and the Lung Cancer Center, ${ }^{\S \S}$ West China Hospital, Sichuan University, Chengdu, PR China; the Department of Bioengineering and Therapeutic Sciences and Liver Center, ${ }^{\dagger}$ University of California, San Francisco, San Francisco, California; the Department of Thoracic Oncology II, ${ }^{\ddagger}$ Key Laboratory of Carcinogenesis and Translational Research, Ministry of Education, Peking University Cancer Hospital and Institute, Beijing, PR China; the Institute of Pathology, ${ }^{\S}$ University of Regensburg, Regensburg, Germany; the Department of Thyroid and Breast Surgery, "The 960th Hospital of the PLA, Jinan, PR China; the Department of Pediatrics," Zhongnan Hospital of Wuhan University, Wuhan, PR China; the Second Department of Pathology, ** Semmelweis University, Budapest, Hungary; the Key Laboratory of Biorheological Science and Technology, ${ }^{\dagger \dagger}$ Ministry of Education, College of Bioengineering, Chongqing University, Chongqing, PR China; the Department of Medical, Surgical, and Experimental Sciences, ${ }^{\ddagger \ddagger}$ University of Sassari, Sassari, Italy; and the Department of Pathology, "था University of Pittsburgh School of Medicine, Pittsburgh, Pennsylvania

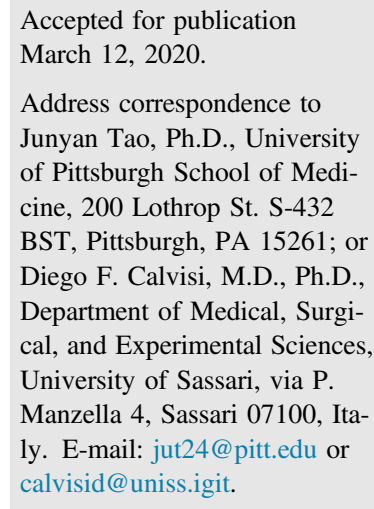

Hepatoblastoma (HB) is the most common pediatric liver tumor. Though Wnt/ $\beta$-catenin and Hippo cascades are implicated in HB development, studies on crosstalk between $\beta$-catenin and Hippo downstream effector transcriptional coactivator with PDZ-binding motif (TAZ) in HB are lacking. Expression levels of TAZ and $\beta$-catenin in human HB specimens were assessed by immunohistochemistry. Functional interplay between TAZ and $\beta$-catenin was determined by overexpression of an activated form of TAZ (TAZS89A), either alone or combined with an oncogenic form of $\beta$-catenin $(\Delta \mathrm{N} 90-\beta$-catenin), in mouse liver via hydrodynamic transfection. Activation of TAZ often co-occurred with that of $\beta$-catenin in clinical specimens. Although the overexpression of TAZS89A alone did not induce hepatocarcinogenesis, concomitant overexpression of TAZS89A and $\triangle \mathrm{N} 90-\beta$-catenin triggered the development of HB lesions exhibiting both epithelial and mesenchymal features. Mechanistically, TAZ/ $\beta$-catenin-driven HB development required TAZ interaction with transcriptional enhanced associate domain factors. Blockade of the Notch cascade did not inhibit TAZ/ $\beta$-catenin-dependent HB formation in mice but suppressed the mesenchymal phenotype. Neither Yes-associated protein nor heat shock factor 1 depletion affected HB development in TAZ/ $\beta$ catenin mice. In human HB cell lines, silencing of TAZ resulted in decreased cell growth, which was further reduced when TAZ knockdown was associated with suppression of either $\beta$-catenin or Yes-associated protein. Overall, our study identified TAZ as a crucial oncogene in HB development and progression. (Am J Pathol 2020, 190: 1397-1413; https://doi.org/10.1016/j.ajpath.2020.03.011)
Hepatoblastoma (HB) is a rare type of cancer, affecting approximately 1 to 2 per million individuals. ${ }^{1}$ It is the most common type of pediatric liver cancer, and mainly occurs in children under 5 years of age. Recently, studies from the Children's Hepatic Tumors International Collaboration have stratified the staging of HB based on clinical information. ${ }^{2}$ These studies established the guidelines for selecting the best treatment for $\mathrm{HB}$ patients. For primary $\mathrm{HB}$, for instance, surgical resection remains the best and most radical therapy for HB. Furthermore, cisplatin-based treatment is the standard chemotherapeutic strategy, and has

Supported by Italian Association Against Cancer (AIRC) grant IG19175 (D.F.C.); NIH grant R01CA204586 (X.C.); National Natural Science Foundation of China grants 81573024 (F.X.), 81802774 (P.L.), and 81702448 (X.L.); and UCSF Liver Center grant P30DK026743 (X.C.).

Disclosures: None declared. 
been used in both adjuvant and neoadjuvant settings in $\mathrm{HB}$ patients. ${ }^{3}$ While the long-term survival rate in children with localized HB is excellent, some patients are diagnosed with metastatic HB or have relapsed disease. These patients exhibit a poor prognosis, with a 5-year survival rate of $30 \%$ to $40 \%$. $^{3,4}$

HB is considered to originate from primary hepatoblasts and/or hepatic stem cells, as a consequence of a failure of these cells to properly differentiate into mature hepatocytes. ${ }^{4}$ Histologically, HB is classified into two major subgroups: epithelial and mixed epithelial/mesenchymal HB. The epithelial subtype can exhibit the phenotype of fetal, embryonal, combined fetal/embryonal, or undifferentiated small cells. HBs in the mixed subtype display mesenchymal cells within the tumor lesions. ${ }^{5}$ This HB subtype is associated with aggressive growth, invasion, and resistance to chemotherapy.

Over the past few years, the application of transcriptomic and sequencing technologies to human $\mathrm{HB}$ has provided mechanistic insights into the molecular pathogenesis of this pediatric malignancy. In particular, accumulating evidence supports the activation of the $\mathrm{Wnt} / \beta$-catenin pathway as the driver oncogenic event in HB development. Indeed, oncogenic/activating mutations of the CTNNB1 gene, which encodes $\beta$-catenin protein, occur in $60 \%$ to $80 \%$ of human HB samples. ${ }^{4}$ Most of the mutations are $\mathrm{N}$-terminal deletions or point mutations in exon 3 of the CTNNB1 gene, leading to the stabilization and nuclear localization $\beta$-catenin. Once in the nucleus, $\beta$-catenin binds to T-cell factor/ lymphoid enhancer factor transcription factors, with consequent induction of downstream effectors, including MYC proto-oncogene (c-myc), cyclin D1, and glutamine synthase (GS or GLUL), which have all been implicated in tumor development. For instance, in a recent study, it was revealed that GLUL maintains mammalian target of rapamycin complex 1 activation downstream of activated Wnt/ $\beta$-catenin, underlying a novel cell intrinsic regulation of the mammalian target of rapamycin complex 1 cascade by the Wnt/ $\beta$-catenin pathway. ${ }^{7}$ Besides activating $C T N N B 1$ mutations, inactivating mutations of $A P C$, a major negative regulator of the $\mathrm{Wnt} / \beta$-catenin cascade, also occur in human $\mathrm{HB},{ }^{8}$ further supporting the pivotal role of the Wnt $/ \beta$-catenin pathway in this disease.

While activation of the Wnt/ $\beta$-catenin cascade has been recognized as a major oncogenic event in $\mathrm{HB}$, this alteration alone has been proved to be insufficient for promoting $\mathrm{HB}$ formation in vivo. Consequently, it has been suggested that a second hit is required for inducing $\mathrm{HB}$ development in cooperation with activated $\mathrm{Wnt} / \beta$-catenin. A recent investigation found that activation of Yes-associated protein (YAP), a downstream effector of the Hippo tumor suppressor pathway, might represent a second important oncogenic signal in HB. ${ }^{9}$ The Hippo signaling is an evolutionary conserved cascade that regulates cell size, metabolism, and growth. ${ }^{10}$ In the liver, the Hippo pathway is implicated in modulating liver regeneration, fibrosis, and tumor development. ${ }^{11}$ In mammalian cells, Hippo kinases, namely macrophage-stimulating proteins 1 and 2 , phosphorylate and activate large tumor suppressor 1 and 2 kinases, which subsequently phosphorylate YAP and its paralog transcriptional coactivator with PDZ-binding motif (TAZ). The phosphorylation of YAP and TAZ proteins results in their cytoplasm translocation and degradation. ${ }^{10}$ When the Hippo cascade is instead suppressed, YAP and TAZ are unphosphorylated and, therefore, activated. After activation, YAP and TAZ localize to the nucleus, where they interact with transcriptional enhanced associate domain (TEAD) DNA-binding proteins, leading to the increased expression of their target genes, such as connective tissue growth factor ( $C C N 2$, alias CTGF), cysteine-rich heparinbinding protein 61 (CCN1, alias CYRR61), and baculoviral inhibitor of apoptosis repeat-containing 5 (BIRC5 or SURVIVIN $).{ }^{10}$ Previously, we showed the coordinated activation of YAP and $\mathrm{Wnt} / \beta$-catenin in about $80 \%$ of human $\mathrm{HB}$ samples. ${ }^{9}$ In vitro, silencing of YAP inhibited the growth of HB cell lines. ' Importantly, co-expression of activated forms of YAP (YAPS127A) and $\beta$-catenin $(\Delta$ N90- $\beta$-catenin or $\beta$-cateninS45Y) synergizes to promote HB formation in mice (YAPS127A/ $\beta$-catenin). ${ }^{9,12}$ Mechanistically, TEAD4 is the major TEAD family member in mouse and human HBs, where it mediates YAPS127A/ $\beta$-catenin-driven $\mathrm{HB}$ formation. ${ }^{13}$

TAZ/WW domain containing transcription regulator 1 is a paralog of YAP, and has been found to possess both distinct and redundant roles with YAP in tumorigenesis. ${ }^{14}$ While previous studies have focused on YAP in HB molecular pathogenesis, the functional role of TAZ in this tumor type has not been defined to date. ${ }^{9}$ In this study, we systematically investigated the expression and functions of TAZ in HB using a comprehensive approach consisting in the analysis of human HB samples, HB cell lines, and mouse models. Our data indicate that TAZ plays an oncogenic role in $\mathrm{HB}$ in cooperation with $\beta$-catenin. In this tumor type, TAZ possesses functions common to and distinct from those in YAP.

\section{Materials and Methods}

\section{Human HB Samples}

Formalin-fixed, paraffin-embedded tissues from a collection of 32 human HB samples were used for the study. The HB specimens were collected at the Department of Pathology, Semmelweis University (Budapest, Hungary) and the Institute of Pathology, Medical University of Regensburg (Regensburg, Germany). Clinicopathologic features of the human HB samples are reported in Table 1. All subjects or their parents/guardians provided informed consent/assent for inclusion in the investigation. The study was performed with the institutional review board approval of the local ethics committee of the Medical University of Regensburg (approval code 17-1015-101, July 4, 2018) and the regional 
Table 1 Clinicopathologic Features of Human Hepatoblastoma Patients

\begin{tabular}{ll}
\hline Variables & Value \\
\cline { 2 - 2 } Patients, $n$ & 32 \\
\hline Male & 18 \\
$\quad$ Female & 14 \\
Age, means \pm SD, years & $4.0 \pm 2.6$ \\
Tumor morphology, $n$ & \\
$\quad$ Fetal & 14 \\
Embryonal & 8 \\
Mixed & 8 \\
Small undifferentiated & 2 \\
Recurrence, $n$ & \\
Yes & 12 \\
No & 20 \\
Lung metastases, $n$ & \\
Yes & 10 \\
No & 22 \\
\hline
\end{tabular}

ethics committee of Semmelweis University (approval code TUKEB 35/2011, March 17, 2011).

\section{Plasmids and Reagents}

The plasmids used in this study, including pT3-EF1 $\alpha$ $\Delta$ N90- $\beta$-catenin, $\quad$ pT3-EF1 $\alpha$-TAZS89A, pT3-EF1 $\alpha$ dnRBP-J, pT3-EF1 $\alpha$-dnHSF1, pCMV-Cre, pCMV empty vector, and $\mathrm{pCMV} / \mathrm{sleeping}$ beauty transposase, have been previously described in detail. ${ }^{9,13}$ The constitutively active form of TAZ (TAZS89A) was purchased from Addgene (Cambridge, MA; plasmid 24815) and the TEAD-binding deficient form of TAZ (TAZS89AS51A) was site-mutated using PCR; the two genes were then cloned into the pT3-EF1 $\alpha$ plasmid (pT3-EF1 $\alpha$-TAZS89AS51A) via Gateway cloning technology (InvitroGen, Carlsbad, CA). All of the plasmids for hydrodynamic injection were purified with the Endotoxin-Free Maxiprep kit (Sigma-Aldrich, St. Louis, MO). The pLKO.1 plasmid (empty vector control) was purchased from Addgene (catalog number 10879).

\section{Hydrodynamic Tail Vein Gene Delivery}

FVB/N mice were purchased from The Jackson Laboratory (Bar Harbor, ME), while Yap $^{\text {flox/flox }}$ conditional knockout mice were kindly provided by Dr. Eric Olson (University of Texas Southwestern Medical Center, Dallas, TX). ${ }^{15}$ The hydrodynamic injection procedure has been described in detail in previous studies. ${ }^{16-19}$ Briefly, to determine the oncogenic potential of TAZS89A, $20 \mu \mathrm{g}$ of pT3-EF1 $\alpha-$ TAZS89A, either alone or in combination with $20 \mu \mathrm{g}$ pT3EF1 $\alpha-\Delta$ N90- $\beta$-catenin, were mixed together with pCMV/ sleeping beauty transposase at a ratio of $25: 1$ and injected into 6- to 8-week-old FVB/N mice via the lateral tail vein. To investigate whether the oncogenic potential of TAZ is based on TAZ transcriptional function, $20 \mu \mathrm{g}$ of pT3-EF1 $\alpha-$
TAZS89AS51A was introduced into adult FVB/N mice along with $20 \mu \mathrm{g}$ pT3-EF1 $\alpha-\Delta$ N90- $\beta$-catenin and $1.6 \mu \mathrm{g}$ $\mathrm{pCMV} /$ sleeping beauty transposase. To block the Notch cascade, high doses of dominant negative form of recombination signal binding protein for immunoglobulin $\kappa \mathrm{J}$ region $($ dnRBP-J) $(60 \mu \mathrm{g})$ together with pT3-EF1 $\alpha$-TAZS89A (20 $\mu \mathrm{g})$ and pT3-EF $1 \alpha-\Delta \mathrm{N} 90-\beta$-catenin $(20 \mu \mathrm{g})$ plasmids were injected. In addition, to assess the importance of heat shock factor transcription factor (HSF)-1 in TAZ/ $\beta$-catenin-dependent $\mathrm{HB}$ formation, $20 \mu \mathrm{g}$ of $\mathrm{pT} 3-\mathrm{EF} 1 \alpha-$ TAZS89A and $20 \mu \mathrm{g}$ of pT3-EF $1 \alpha-\Delta \mathrm{N} 90-\beta$-catenin were injected together with $60 \mu \mathrm{g}$ of either pT3-EF1 $\alpha$-dnHSF1 or pCMV empty vector. Finally, to study whether YAP is required for TAZ-dependent $\mathrm{HB}$ development, $20 \mu \mathrm{g}$ of pT3EF $1 \alpha$-TAZS89A and $20 \mu \mathrm{g}$ of pT3-EF $1 \alpha-\Delta$ N90- $\beta$-catenin, were injected with $60 \mu \mathrm{g}$ of either pCMV-Cre or pCMV empty vector, as well as $\mathrm{pCMV/sleeping} \mathrm{beauty} \mathrm{transposase}$ into adult $\mathrm{Yap}^{\text {flox/flox }}$ conditional knockout mice. All mice used in the experiments were monitored continually and euthanized at specific time points, as indicated in the main text, or when they became moribund. Mice were maintained and monitored in accordance with protocols approved by the Committee for Animal Research at the University of California, San Francisco (San Francisco, CA).

\section{Histology, Immunohistochemistry, Assessment of Proliferation, Apoptosis, and Microvessel Density}

Liver specimens were harvested and fixed in $10 \%$ formalin overnight at $4^{\circ} \mathrm{C}$ and embedded in paraffin. Staining with hematoxylin and eosin (Fisher Chemical Harris Modified Method Hematoxylin Stains; catalog number SH26-500D; Eosin Y, catalog number 23-314-630; Thermo Fisher Scientific, Waltham, MA) was conducted using a standard protocol on human and mouse liver sections, and slides were analyzed by two expert liver pathologists (M.E. and K.E.) in accordance with the criteria described previously., ${ }^{9,20}$ Immunohistochemistry (IHC) was performed according to our previous studies. ${ }^{9,13}$ TAZ nuclear staining of biliary epithelial cells in the surrounding non-neoplastic liver was used as internal positive control of the staining reaction in each specimen analyzed. Proliferation index was determined in mouse HB lesions by counting Ki-67-positive cells on at least 3000 tumor cells per mouse sample. Apoptosis index was determined in mouse hepatocellular carcinoma lesions by counting terminal deoxynucleotidyl transferase dUTP nick end labeling-positive cells on at least 3000 tumor cells per mouse using the TumorTACS in Situ Apoptosis Detection Kit (Trevigen, Gaithersburg, MD), following the manufacturer's instructions. Assessment of microvessel density (MVD) was conducted as previously reported. ${ }^{21}$ Specifically, liver tumor samples from YAPS127A/ $\Delta \mathrm{N} 90-\beta$-catenin and TAZS89A/ $\Delta$ N90- $\beta$-catenin mice were subjected to immunostaining with the mouse monoclonal anti-CD34 antibody (Abcam, Cambridge, UK). The tumors were first screened at low power $(\times 40)$ to identify the areas of highest MVD. The 
four highest MVD areas for each tumor were photographed at high power $(\times 200)$ and the size of each area standardized using the ImageJ software version 1.46 (NIH, Bethesda, MD; https://imagej.nih.gov/ij). Any brown-stained endothelial cell or endothelial cell cluster was counted as one microvessel, irrespective of the presence of a vessel lumen. MVD was expressed as the percentage (means $\pm \mathrm{SD}$ ) of the total CD34-stained spots per section area $\left(0.94 \mathrm{~mm}^{2}\right)$. The primary antibodies used in the study are described in Table 2.

\section{Western Blot Analysis}

Frozen liver tissues and cell pellets were homogenized in Mammalian Protein Extraction Reagent (Thermo Fisher Scientific) containing the phosphatase inhibitors. Protein concentration was determined using a Bio-Rad protein assay kit (Bio-Rad, Hercules, CA) using bovine serum albumin as a standard. Supernatants were denatured by boiling in Tris-Glycine SDS Sample Buffer (Life Technologies, Carlsbad, CA) for Western blot analysis. Proteins were separated by SDS-PAGE and transferred onto nitrocellulose membranes (Life Technologies) by electroblotting. Membranes were blocked in 5\% nonfat dry milk for 1 hour and then incubated with proper primary antibodies (Table 2). Subsequently, membranes were incubated with horseradish peroxidase-secondary antibodies (Jackson ImmunoResearch Laboratories Inc., West Grove, PA) diluted 1:5000 for 30 minutes. Proteins bands were revealed with the Super Signal West Femto (Pierce Chemical Co., New York, NY).

\section{RNA Extraction and Quantitative Real-Time RT-PCR}

Total mRNA from liver tissues and cells was extracted by using the Quick RNA Miniprep Kit (Zymo Research, Irvine, CA).

Table 2 Primary Antibodies Used for Immunohistochemistry and Western Blot Analysis

\begin{tabular}{|c|c|c|c|c|}
\hline Antibody name & Use & Concentration & Company & Catalog no. \\
\hline CK7 & $\mathrm{IHC}$ & $1: 400$ & Abcam & Ab181598 \\
\hline Ki-67 & $\mathrm{IHC}$ & $1: 100$ & Bethyl Laboratories (Montgomery, TX) & IHC-00375 \\
\hline E-cadherin & IHC & $1: 100$ & Cell Signaling Technology (Beverly, MA) & 3195 \\
\hline Myc-tag & IHC; WB & $1: 100 ; 1: 1000$ & MMCRI (Scarborough, ME) & Vli01 \\
\hline V5-tag & IHC & $1: 100$ & Abcam & Ab95038 \\
\hline Yap & IHC; WB & $1: 100 ; 1: 1000$ & Cell Signaling Technology & 14074 \\
\hline$\alpha$-SMA & $\mathrm{IHC}$ & $1: 400$ & Abcam & Ab5994 \\
\hline TAZ/WWTR1 & $\mathrm{IHC}$ & $1: 100$ & Sigma-Aldrich (St. Louis, M0) & AMab90730 \\
\hline TAZ & $\mathrm{IHC}$ & 1:100 & BD Biosciences (San Jose, CA) & 560235 \\
\hline $\mathrm{CEBP} \alpha$ & $\mathrm{IHC}$ & $1: 100$ & Cell Signaling Technology & 8178 \\
\hline GLUL & $\mathrm{IHC}$ & $1: 500$ & BD Biosciences & 610517 \\
\hline CD44v6 & $\mathrm{IHC}$ & $1: 100$ & Thermo Fisher Scientific (Waltham, MA) & MA1-81995 \\
\hline Nanog & $\mathrm{IHC}$ & $1: 100$ & Abcam & Ab214549 \\
\hline CD10 & $\mathrm{IHC}$ & $1: 100$ & Abcam & Ab256494 \\
\hline GATA4 & $\mathrm{IHC}$ & $1: 100$ & Abcam & Ab84593 \\
\hline EPCAM & $\mathrm{IHC}$ & $1: 100$ & Abcam & Ab221552 \\
\hline ERG & $\mathrm{IHC}$ & $1: 100$ & Abcam & Ab92513 \\
\hline CD34 & $\mathrm{IHC}$ & $1: 150$ & Abcam & Ab8158 \\
\hline NCAM1/CD56 & $\mathrm{IHC}$ & $1: 400$ & Cell Signaling Technology & 99746 \\
\hline HSF1 & IHC & $1: 100$ & Cell Signaling Technology & 12972 \\
\hline
\end{tabular}

CEBP, CCAAT/enhancer-binding protein; CK, cytokeratin; EPCAM, epithelial cell adhesion molecule; ERG, erythroblast transformation-specific transcription factor; FOX, forkhead box protein; GAPDH, glyceraldehyde phosphate dehydrogenase; GLUL, glutamine synthetase; Hnf, hepatocyte nuclear factor; HSF, heat shock factor protein; IHC, immunohistochemistry; MMCRI, Maine Medical Center Research Institute; Nanog, homeobox protein NANOG; NCAM, neural cell adhesion molecule; PDGFR, platelet-derived growth factor receptor; SMA, smooth muscle actin; WB, Western blot; WWTR, WW domain-containing transcription regulator protein. 
Next, mRNA expression levels of the genes of interest were detected by real-time quantitative RT-PCR (RT-qPCR) using validated gene expression assays for human and mouse genes (Thermo Fisher Scientific) (Table 3). PCR reactions were performed with $100 \mathrm{ng}$ of cDNA of the collected samples or cell lines, using an ABI Prism 7000 Sequence Detection System with TaqMan Universal PCR Master Mix (Applied Biosystems/ AB Sciex, Foster City, CA). Cycling conditions were as follows: denaturation at $95^{\circ} \mathrm{C}$ for 10 minutes, 40 cycles at $95^{\circ} \mathrm{C}$ for 15 seconds, and then extension at $60^{\circ} \mathrm{C}$ for 1 minute. Quantitative values were calculated by using the StepOne software version 2.3 (Thermo Fisher Scientific) and expressed as $\mathrm{N}$ target $=2^{-\Delta C_{\tau}}$, wherein the $\Delta C_{\tau}$ value of each sample was calculated by subtracting the mean $C_{\tau}$ value of the target gene from the mean $C_{\tau}$ value of the $\beta$-actin gene.

Table 3 Validated Gene Expression Assays Used for Quantitative Real-Time RT-PCR Experiments

\begin{tabular}{|c|c|}
\hline Name & Catalog no.* \\
\hline Mouse Afp & Mm00431715_m1 \\
\hline Mouse GPC3 & Mm00516722_m1 \\
\hline Mouse Dlk1 & Mm00494477_m1 \\
\hline Mouse Cited1 & Mm01235642_g1 \\
\hline Mouse Epcam & Mm00493214_m1 \\
\hline Mouse Gata4 & Mm00484689_m1 \\
\hline Mouse Nanog & Mm02019550_s1 \\
\hline Mouse Vim & Mm01333430_m1 \\
\hline Mouse Des & Mm00802455_m1 \\
\hline Mouse Axin 2 & Mm00443610_m1 \\
\hline Mouse Glul & Mm00725701_s1 \\
\hline Mouse Tbx3 & Mm01195726_m1 \\
\hline Mouse Ccn2 & Mm01192933_g1 \\
\hline Mouse Ccn1 & Mm00487498_m1 \\
\hline Mouse Jag1 & Mm00496902_m1 \\
\hline Mouse Notch1 & Mm00627185_m1 \\
\hline Mouse Notch2 & Mm00803077_m1 \\
\hline Mouse Hes1 & Mm01342805_m1 \\
\hline Mouse Nrf2 & Mm00477784_m1 \\
\hline Mouse Keap1 & Mm00497268_m1 \\
\hline Mouse Nqo1 & Mm01253561_m1 \\
\hline Mouse Hsf1 & Mm01201402_m1 \\
\hline Mouse $\beta$-actin & Mm00607939_s1 \\
\hline Human $\beta$-catenin (CTNNB1) & Hs99999168_m1 \\
\hline Human TAZ & Hs00210007_m1 \\
\hline Human YAP & Hs00902712_g1 \\
\hline Human CCN2 & Hs00170014_m1 \\
\hline Human HSF1 & Hs00232134_m1 \\
\hline Human CCN1 & Hs00155479_m1 \\
\hline Human NOTCH2 & Hs01050702_m1 \\
\hline Human $\beta$-actin & Hs01060665_g1 \\
\hline
\end{tabular}

*Thermo Fisher Scientific

Afp, $\alpha$-fetoprotein; Cited, Cbp/p300-interacting transactivator; CCN2, connective tissue growth factor; CTNN, catenin; CYR, cysteine-rich angiogenic inducer; Des, desmin; Dlk, protein $\delta$ homolog; Epcam, epithelial cell adhesion molecule; Glul, glutamine synthetase; GPC, glypican; Hes, transcription factor HES; HSF, heat shock factor protein; Jag, protein jagged; Keap, Kelch-like ECH-associated protein; Nanog, homeobox protein Nanog; $\mathrm{Nqo}, \mathrm{NAD}(\mathrm{P}) \mathrm{H}$ quinone dehydrogenase; Nrf, namely nuclear factor erythroid-derived 2, like 2; Tbx, T-box transcription factor; Vim, vimentin.

\section{Cell Lines and in Vitro Study}

The Hep293TT cell line was a generous gift of Dr. Gail Tomlinson (University of Texas Southwestern Medical Center, Dallas, TX). The HepG2 cell line was purchased from ATCC (Manassas, VA). Both cell lines were authenticated (Genetica DNA Laboratories, Burlington, NC) and tested clear of mycoplasma contamination. They were maintained as described in a previous study and cultured at $37^{\circ} \mathrm{C}$ in $5 \% \mathrm{CO}_{2}$ humidified incubator. ${ }^{9,13}$ For knockdown studies in vitro, HepG2 and Hep293TT cell lines were transfected with siRNA against human TAZ (catalog number s24789), $\beta$-catenin (catalog number s436), and/or YAP (catalog number s20366), or scrambled siRNA (catalog number s4390846, negative control) (Thermo Fisher Scientific) using the Lipofectamine RNAiMAX Transfection Reagent (Thermo Fisher Scientific) according to the manufacturer's instructions. Transient transfection experiments using pT3-EF1 $\alpha$-TAZS89A and pT3EF1 $\alpha$-TAZS89AS51A plasmids were conducted in HepG2 and Hep293TT cells using the Lipofectamine 2000 Reagent (Thermo Fisher Scientific) following the manufacturer's protocol. Cell proliferation was assessed in HepG2 and Hep293TT cell lines at the 48-hour time point using the BrdU Cell Proliferation Assay Kit (Cell Signaling Technology, Danvers, MA). Apoptosis was determined in the same cell lines using the Cell Death Detection Enzyme-Linked Immunosorbent Assay Plus Kit (Roche Molecular Biochemicals, Indianapolis, IN), following the manufacturer's instructions. All experiments were repeated at least three times in triplicate.

\section{Statistical Analysis}

All data are presented as means \pm SD for each group. Statistical differences between two groups were determined using the $U$-test embedded in Prism software version 6.0 (GraphPad Software Inc., La Jolla, CA). $P<0.05$ was considered statistically significant.

\section{Results}

\section{Frequent Activation of TAZ in Human HB}

First, the levels of TAZ protein in a collection of human HB specimens $(n=32)$ were assessed by IHC (Figure 1A). In nontumorous tissues surrounding the liver, cytoplasmic and nuclear positive staining for TAZ was limited to biliary cells (which were used as an internal positive control for the staining), whereas hepatocytes showed either absent or faint cytoplasmic immunolabeling for this protein, in complete accordance with previous data. ${ }^{22}$ An equivalent staining pattern was detected for the TAZ paralog, YAP, as well as for NOTCH2, a TAZ target. As expected, $\beta$-catenin protein was expressed in the membrane of hepatocytes as well as in the membrane and cytoplasm of biliary cells. Noticeably, strong cytoplasmic and nuclear immunoreactivity for TAZ was detected in most HB specimens (22/32, 68.8\%). The 


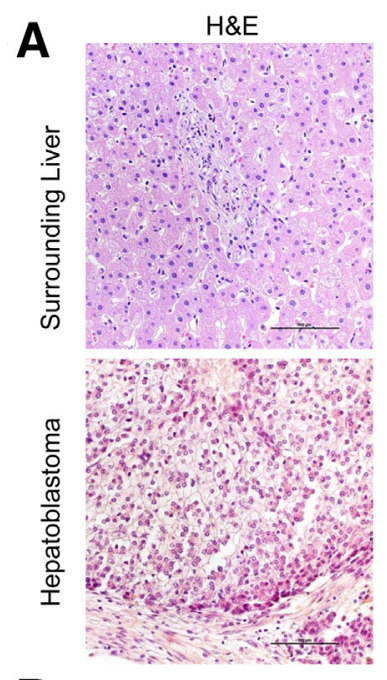

B

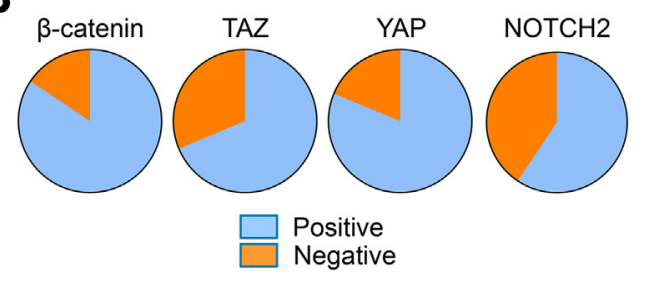

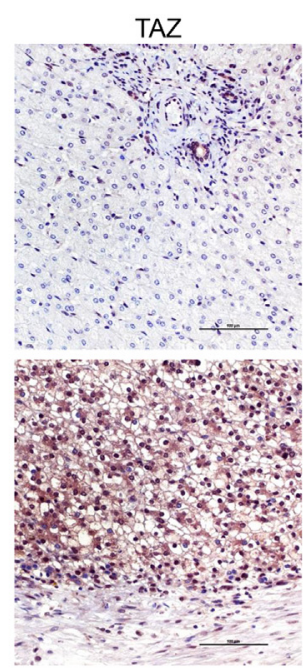
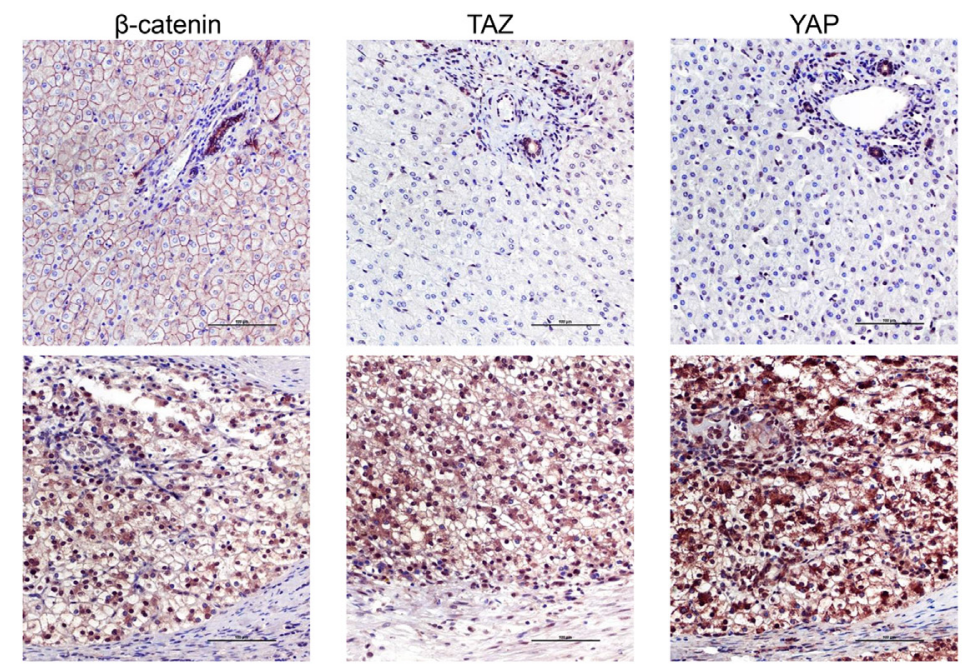

C

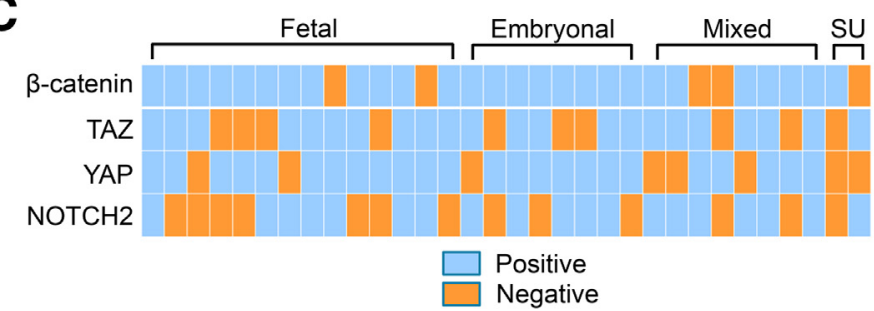

Figure 1 Frequent activation of tafazzin (TAZ) in human hepatoblastoma (HB). A: Representative immunohistochemical patterns of $\beta$-catenin, TAZ, yesassociated protein (YAP), and notch receptor 2 (NOTCH-2) proteins in human normal liver (top row) and HB (fetal subtype; bottom row). B: Positive nuclear staining for $\beta$-catenin, TAZ, YAP, and NOTCH2 proteins in HB patients. C: Distribution of $\beta$-catenin, TAZ, YAP, and NOTCH2 positive nuclear samples in the different HB histopathologic subtypes (a total of $32 \mathrm{HB}$ specimens were analyzed). Scale bars $=100 \mu \mathrm{m}$. Original magnification, $\times 200$. H\&E, hematoxylin and eosin; SU, small undifferentiated.

frequency was lower than that found for nuclear $\beta$-catenin $(27 / 32,84.4 \%)$ and the TAZ paralog YAP $(26 / 32,81.3 \%)$, but higher than nuclear NOTCH2 $(19 / 32,59.4 \%)$ in the same HB collection. Concomitant nuclear immunoreactivity for TAZ and $\beta$-catenin was detected in 18 of 22 TAZ-positive tumors $(81.8 \%)$, while 16 of $22(72.7 \%)$ human HB with TAZ activation displayed positive nuclear immunolabeling for YAP and NOTCH2 proteins (Figure 1B). The specific nuclear immunoreactivity of the various proteins in the various $\mathrm{HB}$ subtypes is summarized (Figure $1 \mathrm{C}$ and Table 4). No significant associations between the levels of TAZ, $\beta$-catenin, YAP, and NOTCH 2 and the clinicopathologic features of the patients, including age, sex, histology subtype, recurrence, or lung metastasis (data not shown), were found.

Overall, the present data unravel the frequent activation of TAZ in human HB, which is frequently associated with the induction/overexpression of YAP, $\beta$-catenin, and $\mathrm{NOTCH} 2$ proteins.

\section{Constitutively Activated TAZ (TAZS89A) and Oncogenic $\beta$-Catenin in the Promotion of HB Development in Mice}

To elucidate the functional role of TAZ in HB pathogenesis, we investigated whether activated TAZ either induces liver carcinogenesis or cooperates with other oncogenes to promote $\mathrm{HB}$ formation in mice. For this purpose, the constitutively activated form of TAZ (TAZS89A) and the $\mathrm{N}$-terminal-deleted, activated mutant form of $\beta$-catenin $(\Delta \mathrm{N} 90-\beta$-catenin $)$, alone or in combination, were transfected in the mouse liver via hydrodynamic tail vein injection (Figure 2A). TAZS89A is a mutant form allowing Taz to escape phosphorylation-mediated degradation and, thus, leading to Taz unrestrained activity. ${ }^{23}$ Overexpression of TAZS89A alone did not lead to liver tumor formation in mice. Indeed, 21 weeks after hydrodynamic injection, all mice appeared to be healthy. Accordingly, on dissection, no tumor nodule was detected. Subsequent histologic evaluation revealed that the mouse liver was completely normal, lacking any histologic alteration (Figure 2, B and C). Nuclear immunoreactivity for Taz was limited to few, single hepatocytes dispersed in the liver parenchyma (Figure 2C); in addition, proliferation was very low or undetectable in TAZS89A-injected mice (data not shown). As previous studies showed that overexpression of $\Delta \mathrm{N} 90-\beta$-catenin alone does not lead to liver tumor development in mice but cooperates with other oncogenes for cancer formation, ${ }^{24-26}$ this $\beta$-catenin mutant form was co-injected with TAZS89A in the mouse liver (TAZS89A/ $\Delta$ N90- $\beta$-catenin mice) (Figure 2A). Noticeably, concomitant overexpression of TAZS89A with $\triangle N 90-\beta$-catenin resulted in rapid liver tumor formation in TAZS89A/ $\Delta$ N90- $\beta$-catenin mice. By 6 weeks after injection, $100 \%$ of the injected mice developed 
Table 4 Specific Nuclear Immunoreactivity of Proteins in Hepatoblastoma (HB)

\begin{tabular}{|c|c|c|c|c|c|}
\hline Sample ID & HB subtype & Beta catenin nuclear staining & TAZ nuclear staining & YAP nuclear staining & Notch2 nuclear staining \\
\hline 1 & Fetal & Yes & Yes & Yes & Yes \\
\hline 2 & Fetal & Yes & Yes & Yes & No \\
\hline 4 & Fetal & Yes & No & Yes & No \\
\hline 5 & Fetal & Yes & No & Yes & No \\
\hline 6 & Fetal & Yes & No & Yes & Yes \\
\hline 9 & Fetal & Yes & Yes & Yes & Yes \\
\hline 10 & Fetal & No & Yes & Yes & No \\
\hline 11 & Fetal & Yes & No & Yes & No \\
\hline 12 & Fetal & Yes & Yes & Yes & Yes \\
\hline 13 & Fetal & No & Yes & Yes & Yes \\
\hline 18 & Embryonal & Yes & Yes & Yes & No \\
\hline 19 & Embryonal & Yes & No & Yes & Yes \\
\hline 20 & Embryonal & Yes & No & Yes & Yes \\
\hline 21 & Embryonal & Yes & Yes & Yes & Yes \\
\hline 22 & Embryonal & Yes & Yes & Yes & No \\
\hline 23 & Mixed & Yes & Yes & No & Yes \\
\hline 24 & Mixed & Yes & Yes & No & Yes \\
\hline 25 & Mixed & No & Yes & Yes & Yes \\
\hline 26 & Mixed & No & No & Yes & No \\
\hline 27 & Mixed & Yes & Yes & No & Yes \\
\hline 28 & Mixed & Yes & Yes & Yes & Yes \\
\hline
\end{tabular}

high liver tumor burden with palpable abdominal mass and were euthanized based on our Institutional Animal Care and Use Committee protocol (Figure 2, B, D, and E).

Microscopically, small clusters of atypical cells were visible as early as $\sim 1.5$ weeks after injection in the livers of TAZS89A/ $\Delta$ N90- $\beta$-catenin mice (Figure $3 \mathrm{~A}$ ). Positive immunoreactivity for $c-m y c-$ tagged $\Delta \mathrm{N} 90-\beta$-catenin and Taz antibodies confirmed that tumor cells originated from the injected plasmids (Figure 3, B and C), while immunolabeling for Ki-67 implied their active proliferation (Figure 3D). The first tumor nodules were visible in TAZS89A/ $\Delta$ N90- $\beta$-catenin mice by 3 weeks after injection (Figure 3E). Tumors quickly occupied nearly completely the liver parenchyma by 6 weeks after injection (Figure 3F).

Histologically, most of the lesions developed in TAZS89A/ $\Delta$ N90- $\beta$-catenin mice consisted of small cells with prominent nuclei, similar to those previously described in YAPS127A/ $\beta$-catenin mice, ${ }^{9,12}$ and resembling human fetal or crowded fetal HB (Figure 3, G and H). Nonetheless, a second, distinct tumor entity was appreciable in the liver parenchyma of TAZS89A/ $\Delta$ N90- $\beta$-catenin mice. Indeed, besides the pure hepatoblast-like lesions, many tumors displayed a mixed phenotype, consisting of an epithelial (hepatoblast) and a mesenchymal component, with the latter being characterized primarily by cells with spindle morphology (Figure 3, I and J). To the best of our knowledge, this is the first reported HB mouse model showing features of epithelial and mesenchymal differentiation. After this interesting observation, we sought to determine the molecular characteristics of the two tumor entities by using IHC (Figure 4). As expected, both types of lesions displayed immunoreactivity for c-myc-tagged $\Delta \mathrm{N} 90-\beta$-catenin and TAZ antibodies, implying their origin from the injected constructs. Increased levels of the injected constructs were confirmed by Western blot analysis (Supplemental Figure S1). In addition, immunoreactivity for hepatocellular markers [hepatocyte nuclear factor $4 \alpha$, forkhead box A1/A2, CCAAT enhancer binding protein $\alpha$, and GS or GLUL] was significantly most pronounced in hepatoblast-like lesions. Furthermore, levels of the progenitor markers CD44v6, NANOG, CD10, and EPCAM, as well as the cholangioblast markers cytokeratin 7 and 19 (CK7 and CK19), were highest in the epithelial 
A

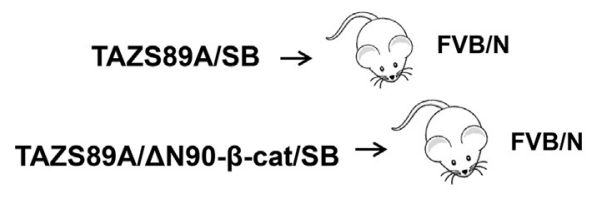

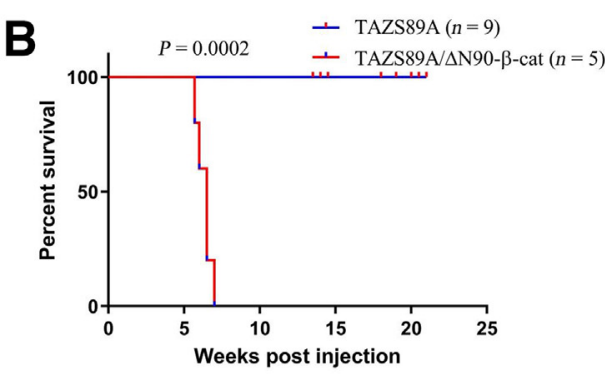

C
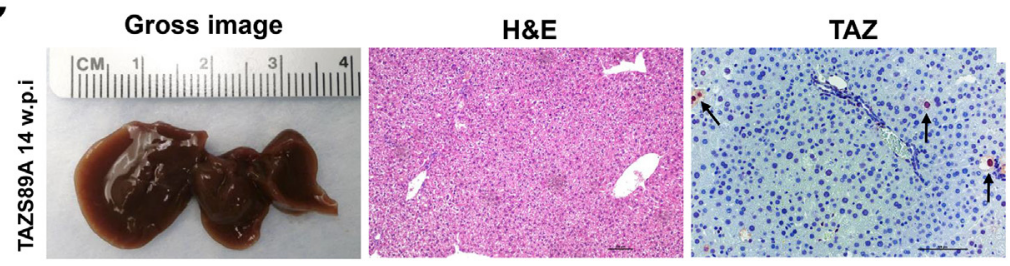

D

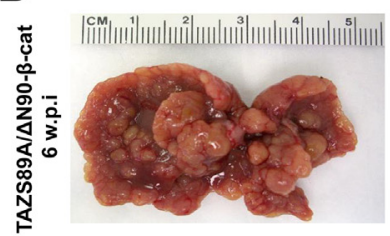

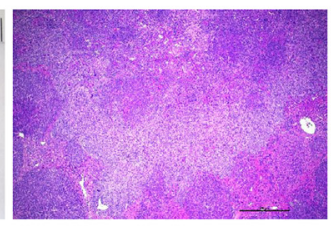

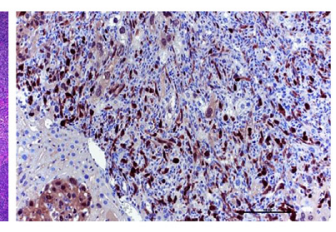

$\mathbf{E}$

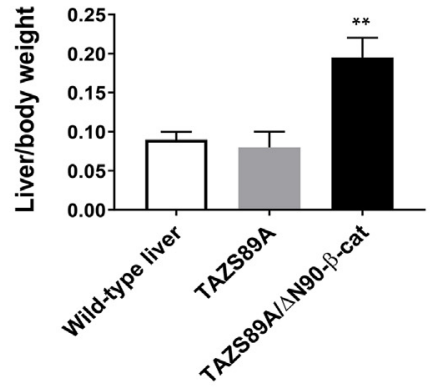

Figure 2 Overexpression of a constitutively activated tafazzin (TAZ) (TAZS89A) form is unable to trigger tumor development alone but cooperates with oncogenic $\beta$-catenin to induce hepatoblastoma (HB) development in mice. A: Scheme of the experiments conducted. B: Survival curves of TAZS89A and TAZS89A/ $\Delta$ N90 - $\beta$-catenin mice. C and D: Gross and microscopic images of TAZS89A and TAZS89A/ $\Delta$ N90 - $\beta$-catenin mouse livers. Note the normal histologic features of the TAZS89A-only injected liver (C), whereas almost the whole parenchyma of TAZS89A/ $\triangle N$ N0 - $\beta$-catenin mice is occupied by tumor lesions (D). While TAZ nuclear immunoreactivity is diffused in the tumor lesions of TAZS89A/ $\Delta$ N90- $\beta$-catenin mice (D), only few, single hepatocytes (arrows) express TAZ in the nucleus of TAZS89A-injected livers (C). E: Liver weight/body weight ratio of normal (wild-type) livers as well as TAZS89A and TAZS89A/ $\triangle \mathrm{N} 90-\beta$-catenin mice. Data are expressed as means \pm SD (E). $n=5$ mice per group. ${ }^{* *} P<0.01$ versus wild-type and TAZS89A mice. Scale bars: $100 \mu \mathrm{m}$ [C, hematoxylin and eosin (H\&E) staining, and C and D, TAZ staining)]; $500 \mu \mathrm{m}$ (D, H\&E staining). Original magnification: $\times 100$ (C, H\&E staining); $\times 200$ (C and D, TAZ staining); $\times 40$ (D, H\&E staining). SB, sleeping beauty transposase; w.p.i., weeks post-injection.

lesions. Levels of the GATA binding factor 4 (GATA4) transcription factor, whose expression is frequently up-regulated in human $\mathrm{HB}$ with mesenchymal features, ${ }^{27}$ were strongly induced in both tumor entities. The mesenchymal part of the lesions, on the other hand, showed the highest levels of stromal- and angiogenesis-related markers, such as ERG, CD34, $\alpha$-smooth muscle actin, CD56/neural cell adhesion molecule 1, platelet-derived growth factor receptor $\beta$, and S100A4, whereas immunoreactivity for the epithelial marker E-cadherin was lost in the same mesenchymal lesions (Supplemental Figure S2).

Furthermore, real-time RT-PCR showed that livers of TAZS89A/ $\Delta$ N90- $\beta$-catenin mice exhibited significantly higher levels than did wild-type livers of glypican $3(G p c 3)$, $\alpha$-fetoprotein (Afp), Citedl, Dlkl, and Gata4 genes (Supplemental Figure S3), whose up-regulation in human $\mathrm{HB}$ is well established. ${ }^{27,28}$ As expected, mRNA expression of Wnt/ $\beta$-catenin [GS (Glul), Axin2, and Tbx3] and Hippo/ TAZ canonical targets [Ccn2, Ccnl, jagged-1 (Jagl), Notch receptor 1 (Notch1), Notch receptor 2 (Notch2), and hairy and enhancer of split-1 (Hes1)] was more elevated in TAZS89A/ $\Delta$ N90- $\beta$-catenin livers when compared with the wild-type counterpart (Supplemental Figure S4).

\section{Interaction of TAZ with TEAD Transcription Factors in} TAZS89A/ $\Delta$ N90- $\beta$-Catenin-Induced HB

TAZ is a transcriptional activator and it interacts with TEAD DNA-binding proteins to induce downstream gene expression. Nonetheless, TAZ also possesses functions that are independent of its binding to TEAD factors. ${ }^{10,11,29}$ Thus, we sought to investigate whether TAZS89A/ $\Delta$ N90- $\beta$-catenin-induced HB development requires an intact TAZ/TEAD interaction. To address this issue, the TAZS89AS51A plasmid was generated for hydrodynamic transfection. Specifically, the S51A mutation prevents TAZ from binding to TEAD proteins. ${ }^{29}$ Therefore, TAZS89AS51A and $\Delta$ N90- $\beta$-catenin plasmids were coexpressed into the mouse liver (Figure 5A). All TAZS89AS51A/ $\Delta$ N90 $-\beta$-catenin mice appeared to have been healthy and were harvested 7 to 11 weeks after injection (Figure 5B). Grossly, no tumors nodules were detected in the mouse liver. Subsequent histologic analysis revealed that the livers of TAZS89AS51A/ $\Delta$ N90- $\beta$-catenin mice were completely normal, and the liver weight/body weight ratio as well as the proliferation index of these mouse livers was similar to that of wild-type, noninjected livers 

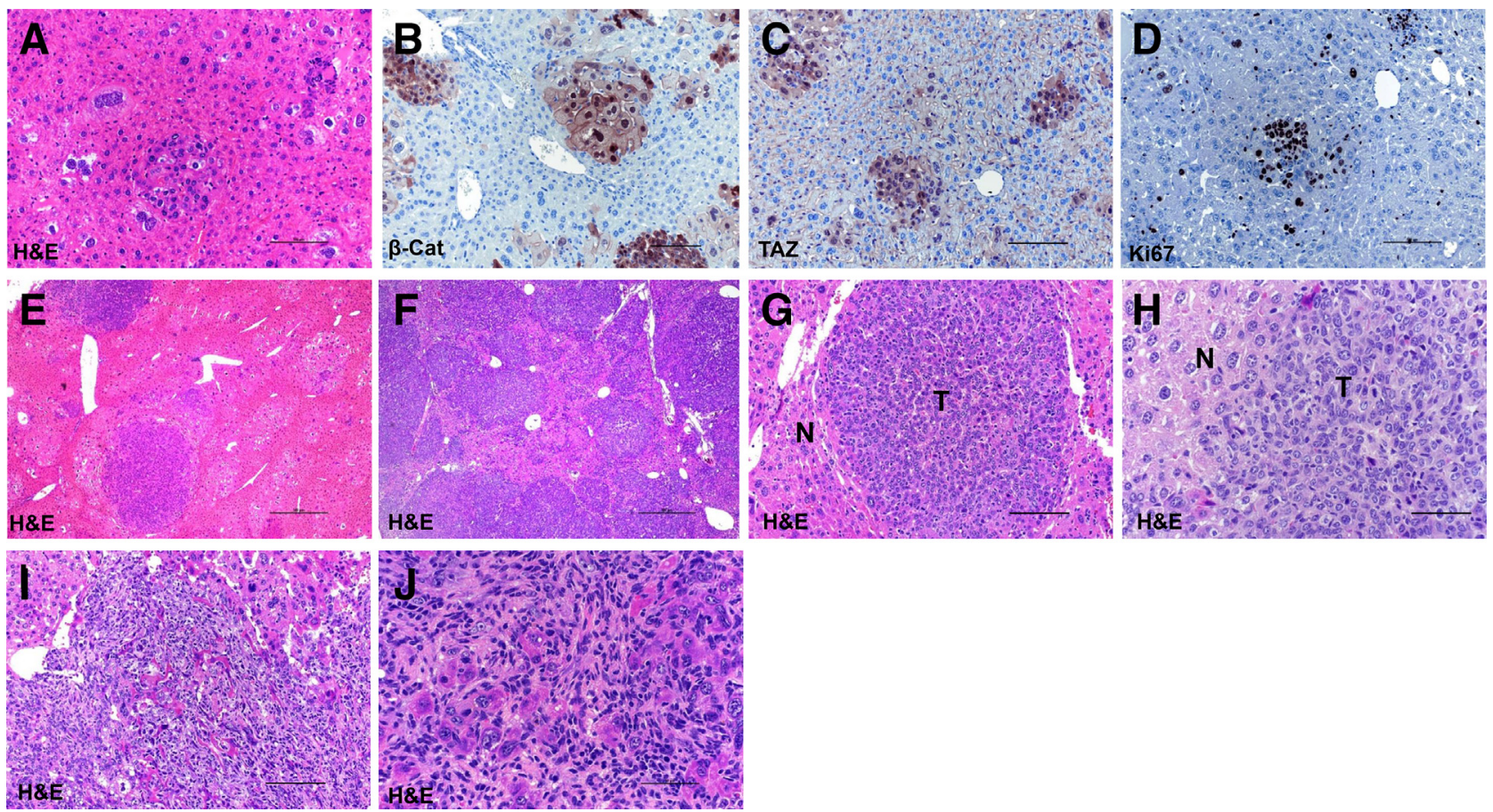

Figure 3 Histopathologic characterization of liver lesions developed in tafazzin (TAZ)S89A/ $\Delta$ N90- $\beta$-catenin mice. A: Hematoxylin and eosin (H\&E) staining of a TAZS89A/ $\triangle N$ N0 $-\beta$-catenin mouse liver 1.5 weeks after hydrodynamic tail vein injection. Altered cells, characterized mainly by nuclei of various size, form little clusters in the liver parenchyma. B-D: These cells express the Myc-tagged $\beta$-catenin (B) and TAZ (C) proteins, indicating their origin from injected constructs, and are actively proliferating (D), as indicated by nuclear immunoreactivity for the proliferation marker Ki-67. E: By 3 weeks after injection, nodules of altered cells are appreciable throughout the liver. These nodular lesions occupy most of the liver surface by 6 weeks after injection. F: Lesions appear, composed of small cells with prominent nuclei and a high nuclear/cytoplasmic ratio, resembling human fetal and crowded fetal hepatoblastomas (HBs). $\mathbf{G}$ and $\mathbf{H}$ : At higher magnification, the small size of tumor cells (T) when compared to normal hepatocytes (N) can be appreciated. I: Intriguingly, together with the epithelial component shown in $\mathbf{F}$, many tumors displayed the presence of a mesenchymal part, characterized by the presence of spindle cells and a stroma component. J: Within the spindle and stromal cells, epithelial/hepatoblast-like cells can be appreciated. Scale bars: $100 \mu \mathrm{m}(\mathbf{A}-\mathbf{D}$, $\mathbf{G}, \mathbf{I}) ; 500 \mu \mathrm{m}(\mathbf{E}$ and $\mathbf{F}) ; 50 \mu \mathrm{m}(\mathbf{H}$ and $\mathbf{J})$. Original magnification: $\times 400(\mathbf{H}$ and $\mathbf{J}) ; \times 200(\mathbf{A}-\mathbf{D}, \mathbf{G}, \mathbf{I}) ; \times 40(\mathbf{E}$ and $\mathbf{F})$.

(Figure 5, C and D). The inability of the TAZS89AS51A plasmid to induce TEAD-mediated downstream effectors was further confirmed in vitro (Supplemental Figure S5). Indeed, forced overexpression of either TAZS89A or TAZS89AS51A triggered equivalent overexpression of TAZ in the human HepG2 and Hep293TT HB cell lines. In striking contrast, transient transfection of TAZS89A but not TAZS89AS51A was able to drive the mRNA upregulation in the same cells of CCN2-, CCN1-, and NOTCH2-specific targets of TAZ, whose induction is mediated by TEAD factors (Supplemental Figure S5, A-D). In addition, mRNA expression of $C c n 2, C c n 1$, and Notch2 were determined in wild-type livers, TAZS89A/ $\Delta \mathrm{N} 90-\beta$-catenin, and TAZS89AS51A/ $\Delta$ N90- $\beta$-catenin liver tissues via quantitative RT-PCR. In accordance with the in vitro data, RT-qPCR showed significant higher mRNA expressions of Ccn2, Ccn1, and Notch2 genes in TAZS89A/ $\Delta N 90-\beta$-catenin mouse liver tissues when compared with those in TAZS89AS51A/ $\Delta$ N90- $\beta$-catenin and wild-type groups, whereas TAZS89AS51A/ $\Delta$ N90- $\beta$ catenin and wild-type liver tissues demonstrated similar levels of these genes (Supplemental Figure S5E). All together, the present data demonstrate that the interaction of Taz with Tead transcription factors is necessary for Tazdriven HB development in mice as well as for the induction of TAZ effectors in vitro.

\section{Commonalities and Differences between TAZS89A/ $\Delta$ N90- $\beta$-Catenin and YAPS127A $/ \Delta N 90-\beta$-Catenin Lesions}

Previous reports showed that the activated form of Yap (YAPS127A), the other oncogenic transducer of the Hippo signaling, cooperates with $\Delta \mathrm{N} 90-\beta$-catenin to induce $\mathrm{HB}$ formation in vivo. ${ }^{9}$ Therefore, liver tumors developed in TAZS89A/ $\Delta$ N90- $\beta$-catenin mice were compared with those from YAPS127A/ $\Delta$ N90- $\beta$-catenin mice. Histologically, the pure hepatoblast-like lesions were indistinguishable between the two models. However, mesenchymal lesions were absent in YAPS127A/ $\Delta$ N90- $\beta$-catenin mice (Supplemental Figure S6A). At the cellular level, the rates of proliferation, apoptosis, and angiogenesis MVD were compared between the epithelial lesions from TAZS89A/ $\Delta \mathrm{N} 90-\beta$-catenin and YAPS127A/ $\Delta \mathrm{N} 90-\beta$-catenin mice. No significant differences were observed in the rates of proliferation and MVD between the two mouse models, 

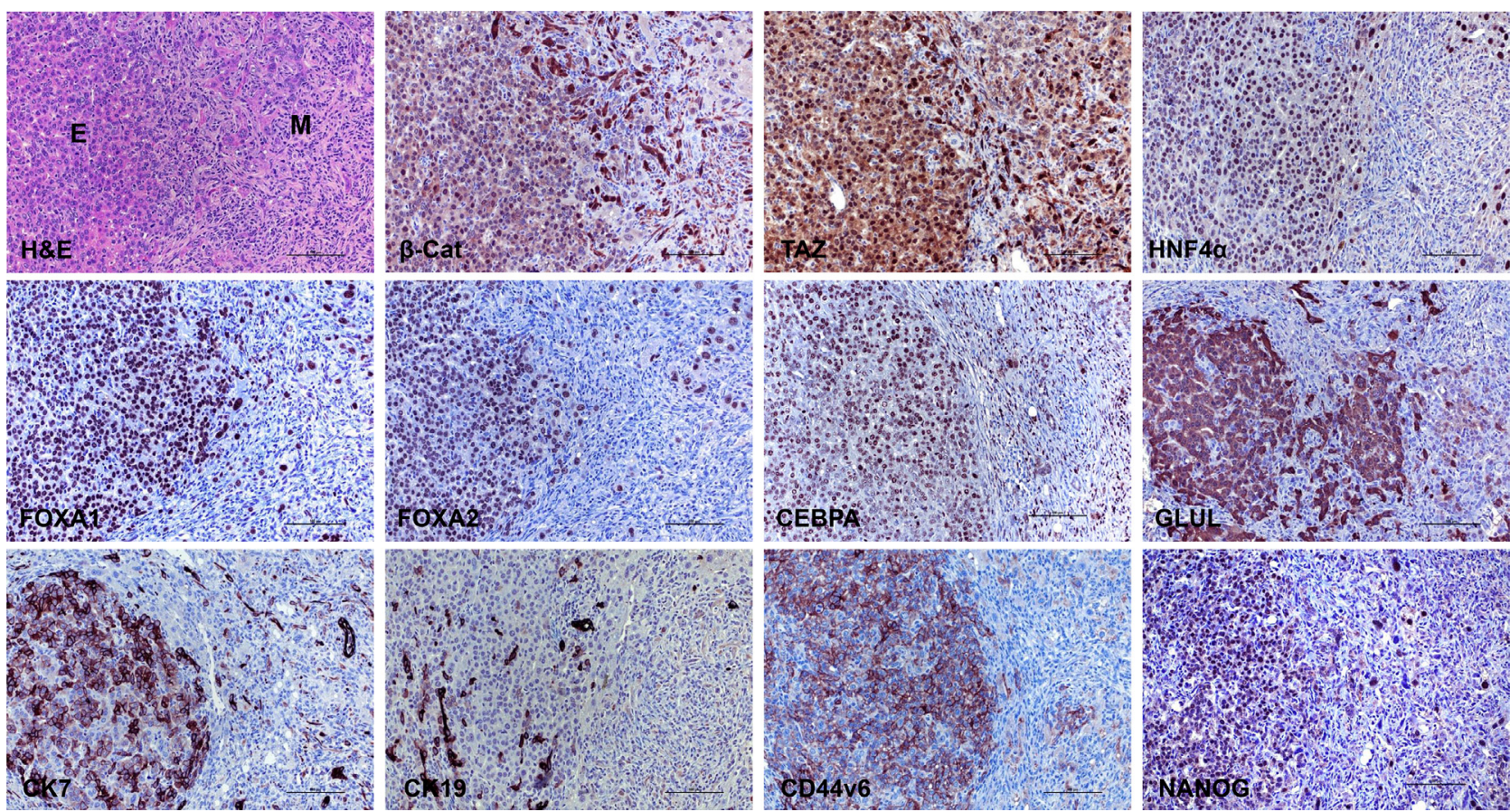

Figure 4 Molecular characterization of liver lesions developed in TAZS89A/ $\Delta$ N90- $\beta$-catenin mice by immunohistochemistry. Staining patterns of the epithelial (E) and the mesenchymal (M) component of TAZS89A/ $\Delta$ N90- $\beta$-catenin induced tumors for several hepatocellular [hepatocyte nuclear factor (HNF)$4 \alpha$, forkhead box protein (FOX)-A1, FOXA2, CCAAT/enhancer-binding protein (CEBP)-A, and glutamine synthetase (GLUL)], cholangiocellular [cytokeratin (CK)7, CK19], and progenitor (CD44v6, homeobox protein NANOG markers are shown in each image. Scale bars $=100 \mu \mathrm{m}$. Original magnification, $\times 200$. H\&E, hematoxylin and eosin.

whereas apoptosis was significantly lower in TAZS89A/ $\Delta$ N90- $\beta$-catenin mice (Supplemental Figure S6, B-D).

At the molecular level, TAZS89A/ $\Delta$ N90- $\beta$-catenin and YAPS127A/ $\Delta$ N90- $\beta$-catenin tumors expressed $A f p$ and $G p c 3$ genes at similar levels. In addition, YAPS127A/ $\Delta \mathrm{N} 90-\beta$-catenin mouse malignant lesions expressed Epcam at higher levels, whereas TAZS89A/ $\Delta$ N90- $\beta$-catenin tumors displayed higher levels of HB markers $D l k l$ and Citedl as well as of the Glul hepatocellular marker. TAZS89A/ $\Delta$ N90- $\beta$-catenin tumors also exhibited the highest mRNA expression of the Gata4 transcription factor. Furthermore, Notch pathway members (Jagl, Notchl, Notch2, and Hes1) exhibited the highest mRNA expression in TAZS89A/ $\Delta$ N90- $\beta$-catenin lesions (Supplemental Figure S7), in accordance with RT-qPCR data. IHC analysis revealed that levels of hepatocellular markers hepatocyte nuclear factor $4 \alpha$, forkhead box a2/a1, and CCAAT enhancer binding protein $\alpha$ (the last two not shown), were similar in the two models. In contrast, Glul protein was expressed homogeneously in TAZS89A/ $\Delta$ N90- $\beta$-catenin malignant lesions, whereas only patchy immunoreactivity for this protein was detected in YAPS127A/ $\Delta$ N90- $\beta$-catenin corresponding lesions. No differences in the levels of the progenitor markers CD44v6 and CD10 or the cholangioblast markers $\mathrm{Ck} 7$ and $\mathrm{Ck} 19$ in the two models were detected, whereas staining intensity for Epcam was significantly stronger and more homogeneous in YAPS127A/ $\Delta$ N90- $\beta$-catenin tumors (Supplemental Figure S8).
Finally, the levels of two transcription factors, whose roles in liver cancer are becoming more and more relevant, namely nuclear factor erythroid-derived 2, like 2 (NRF2) and HSF $1,{ }^{30-32}$ were determined in TAZS89A/ $\Delta$ N90- $\beta$ catenin and YAPS127A/ $\Delta$ N90- $\beta$-catenin tumors. Levels of namely nuclear factor erythroid-derived 2, like 2, its regulator Kelch-like ECH-associated protein 1, and those of its target $\mathrm{NAD}(\mathrm{P}) \mathrm{H}$ quinone dehydrogenase 1 , were upregulated in both mouse models, without statistical differences in the two cohorts compared with wild-type mice (Supplemental Figure S9). In striking contrast, a robust overexpression of HSF1 was observed only in TAZS89A/ $\Delta$ N90- $\beta$-catenin mouse lesions both via RT-qPCR and IHC (Supplemental Figure S9). All together, the present data indicate the existence of common and distinct features between TAZS89A/AN90- $\beta$-catenin and YAPS127A/ $\Delta \mathrm{N} 90-\beta$-catenin tumors.

\section{Role of Canonical Notch Signaling in TAZS89A/} $\Delta \mathrm{N} 90-\beta$-Catenin HB Development and Suppression of the Mesenchymal Component of Mouse Lesions

Mounting evidence indicates the Notch pathway as a downstream effector of the Hippo cascade in liver cancer. $^{33-35}$ In addition, NOTCH2 has been found to be consistently overexpressed in human HB. ${ }^{36}$ Furthermore, our present data indicate a significantly more pronounced up-regulation of members of the Notch pathway 
A

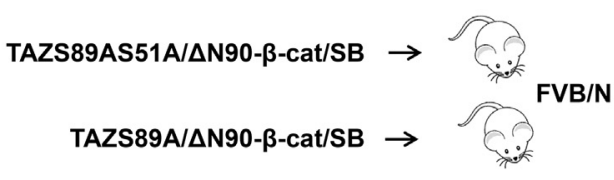

B

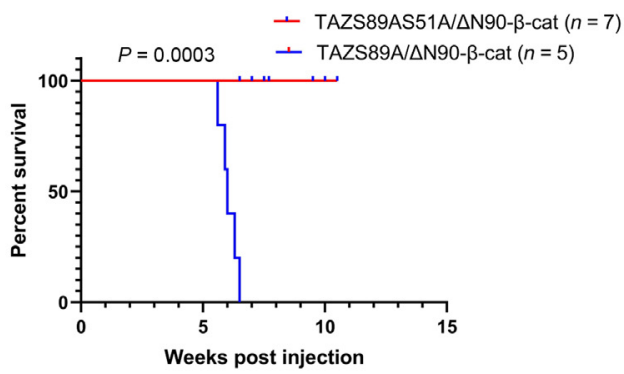

\section{TAZS89AS51A/AN90-B-cat 11 w.p.i}

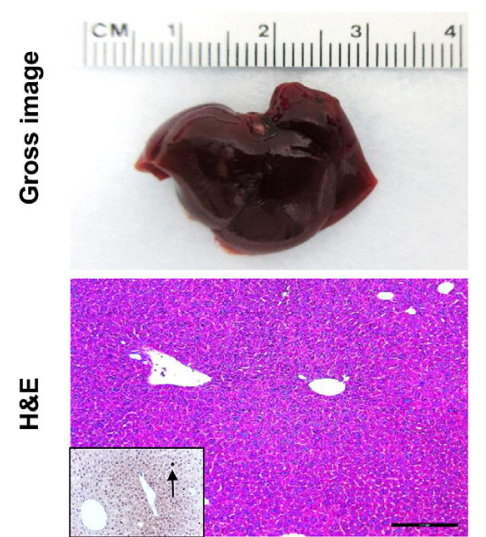

D

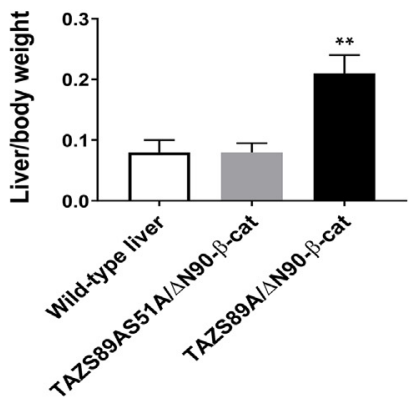

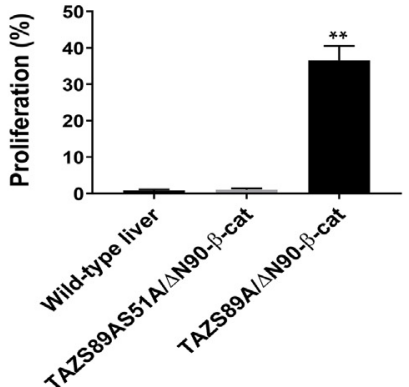

Figure 5 Transcriptionally active tafazzin (TAZ) is required for hepatocarcinogenesis in association with oncogenic $\beta$-catenin. A: Scheme of the experiment. B: Survival curve of TAZS89A/ $\Delta N 90-\beta$-catenin and TAZS89AS51A/ $\Delta N 90-\beta$-catenin mice. TAZS89AS51A overexpression results in the induction of TAZdependent effects that are independent of its binding to transcriptional enhanced associate domain (TEAD) transcription factors. C: Impairment of TAZ binding to TEAD transcription factors by hydrodynamic transfection of the TAZS89AS51A plasmid inhibits TAZS89A/ $\beta$-catenin carcinogenesis in the mouse liver. Histologically, the liver of TAZS89AS51A mice appears indistinguishable from that of wild-type, noninjected livers, and hepatocyte proliferation is extremely low (the arrow in the inset indicates a single Ki-67 positive cell). D: Liver weight/body weight ratio and proliferation index of wild-type as well as TAZS89A/ $\Delta N 90-\beta$-catenin and TAZS89AS51A $/ \Delta N 90-\beta$-catenin mice. Data are expressed as means \pm SD (D). ${ }^{* *} P<0.01$ versus wild-type and TAZS89AS51A/ $\Delta$ N90- $\beta$ catenin mice. Scale bar $=100 \mu \mathrm{m}$. Original magnification: $\times 200$ (C, main image and inset). H\&E, hematoxylin and eosin staining; SB, sleeping beauty transposase.

(Jag1, Notch1, Notch2, and Hes1) in the lesions of TAZS89A/ $\Delta N 90-\beta$-catenin mice than those formed in YAPS127A/ $\triangle$ N90- $\beta$-catenin mice. Thus, we investigated whether the canonical Notch signaling is required for TAZS89A/ $\Delta$ N90- $\beta$-catenin-driven HB formation in vivo. TAZS89A/ $\Delta$ N90 $-\beta$-catenin plasmids were hydrodynamically injected together with dnRBP-J (these mice will be referred to as TAZS89A/ $\Delta$ N90- $\beta$-catenin/dnRBP-J) that has been shown to effectively inhibit the canonical Notch cascade (Figure 6A). ${ }^{35,37}$ Additional mice were injected with TAZS89A/ $\Delta$ N90 $-\beta$-catenin with pT3 empty vector as control (TAZS89A/AN90- $\beta$-catenin/pT3). All mice developed high liver tumor burden and were required to be euthanized around 6 to 9 weeks after injection (Figure 6, B-D). Histologically, HB lesions could be appreciated in both cohorts of mice (Figure 6, C and D). However, overexpression of dnRBP-J in the livers of TAZS89A/ $\Delta$ N90- $\beta$ catenin mice led to the complete disappearance of the mesenchymal compartment of HB lesions (Figure 6C and Supplemental Figure S10), indicating that the Notch pathway significantly contributes to the differentiation toward a mesenchymal phenotype of TAZS89A/ $\triangle \mathrm{N} 90-\beta-$ catenin HB lesions. No overt differences were observed in the TAZS89A/ $\Delta$ N90- $\beta$-catenin and TAZS89A/ $\Delta$ N90 $-\beta$ catenin/dnRBP-J mouse cohorts in terms of liver/body weight ratio (Figure 6E). Similarly, no differences with regard to proliferation and apoptosis were detected between TAZS89A/ $\Delta$ N90- $\beta$-catenin and TAZS89A/ $\Delta$ N90- $\beta$-catenin/dnRBP-J epithelial mouse lesions $(38.5 \pm 4.0$ versus $40.2 \pm 5.2$, respectively; and $12.5 \pm 2.8$ versus $14.0 \pm 3.6$, respectively; $n=5$ per mouse cohort).

Overall, our study indicates that Notch signaling is activated in TAZS89A/DN90- $\beta$-catenin tissues and influences the tumor cell differentiation. However, Notch activation does not inhibit TAZS89A/ $\Delta$ N90- $\beta$-catenin-driven HB development.

\section{HSF1 in TAZS89A $/ \Delta$ N90- $\beta$-Catenin-Dependent Hepatoblastoma Development}

Next, after the interesting finding of up-regulation of HSF1 exclusively in TAZS89A/ $\Delta$ N90- $\beta$-catenin tumors, the levels of HSF1 in human HB specimens were assessed by IHC to determine a possible role of HSF1 in this tumor type. 
A

B
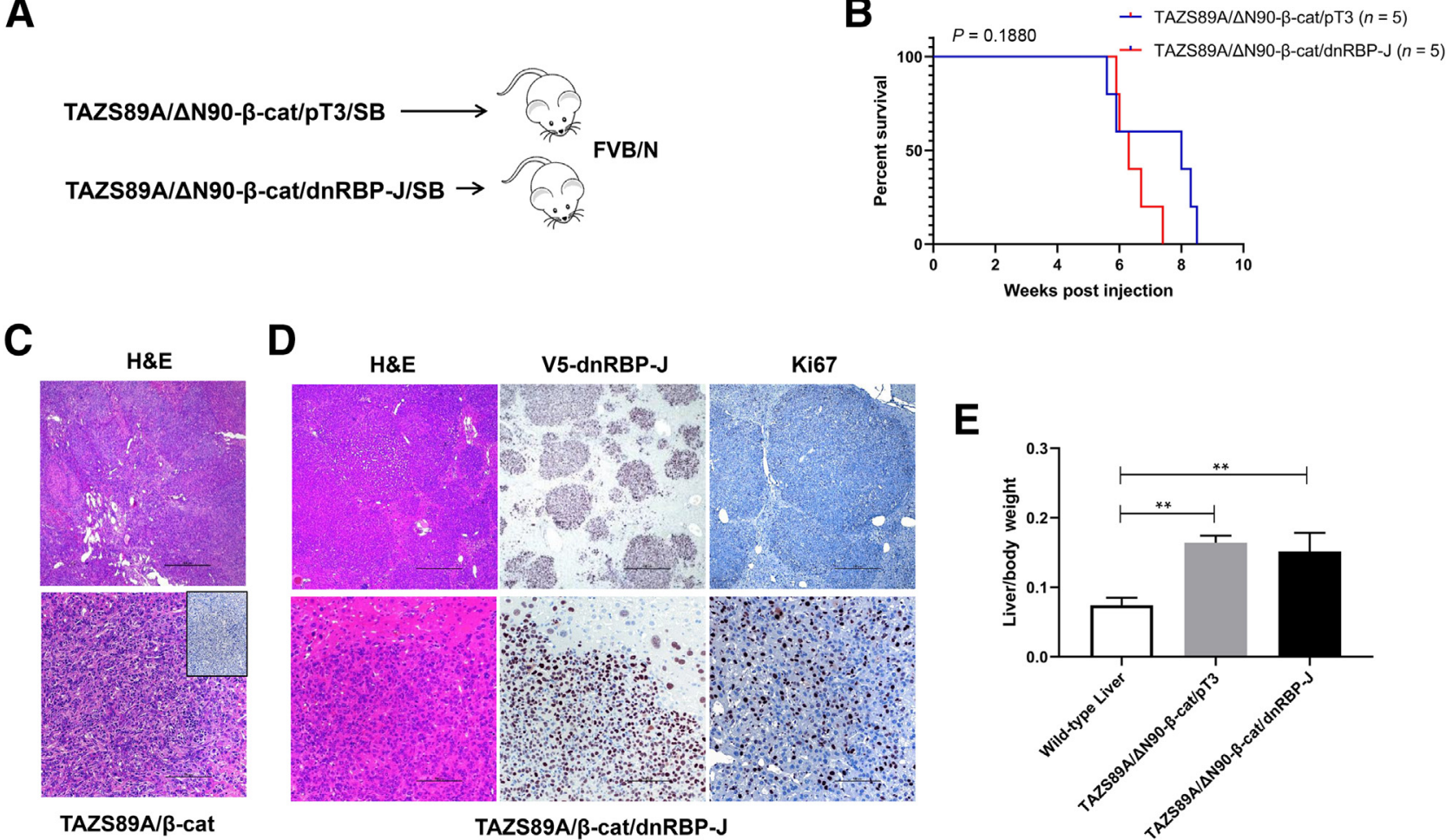

Figure 6 Inactivation of the Notch cascade abolishes the mesenchymal component of liver tumors, without impairing carcinogenesis, in TAZS89A/ $\Delta$ N90- $\beta$-catenin mice. A: Study design. Briefly, wild-type mice were subjected to hydrodynamic tail vein injection of either TAZS89A/ $\Delta$ N90- $\beta$-catenin/pT3 (control) or TAZS89A/AN90- $\beta$-catenin/dnRBP-J plasmid. Specifically, dnRBP-J is the dominant negative form of the transcriptional coactivator recombination signal binding protein for immunoglobulin $\mathrm{\kappa} \mathrm{J}$ region, whose overexpression effectively inhibits the canonical Notch cascade. B: Survival curve of TAZS89A/ $\Delta \mathrm{N} 90-\beta$-catenin/pT3 (control) or TAZS89A/ $\Delta$ N90 $-\beta$-catenin/dnRBP-J mice. C: Liver lesions from TAZS89A $/ \Delta N 90-\beta$-catenin mice are characterized both by an epithelial and a mesenchymal component. D: In contrast, the lesions from TAZS89A/ $\Delta$ N90- $\beta$-catenin/dnRBP-J mice consist only of an epithelial component. Homogeneous immunohistochemical staining for the V5-tagged dnRBP-J construct throughout the lesions indicates the effective transfection of the dnRBP-J plasmid in the tumor cells. These cells are highly proliferative, as indicated by pronounced immunoreactivity for the Ki-67 proliferation marker. As expected, V5 immunoreactivity is completely negative in TAZS89A/ $\Delta \mathrm{N} 90-\beta$-catenin/pT3 mice (C, inset). E: Liver weight/body weight ratio of wild-type as well as TAZS89A/ $\Delta$ N90- $\beta$-catenin/pT3 (control) or TAZS89A/ $\Delta$ N90- $\beta$-catenin/dnRBP-J mice. Data are expressed as means \pm SD (E). ${ }^{* *} P<0.01$ versus wildtype mice. Scale bars: $500 \mu \mathrm{m}$ (C, top panel, and $\mathbf{D}$, top row); $100 \mu \mathrm{m}$ (C, bottom panel, and $\mathbf{D}$, bottom row). Original magnification, $\times 40$ (inset in $\mathbf{C}$ ). H\&E, hematoxylin and eosin staining; SB, sleeping beauty transposase.

Of note, HSF1 was ubiquitously $(32 / 32,100 \%)$ up-regulated and localized in the nucleus of $\mathrm{HB}$ cells in the collection examined (Supplemental Figure S11), whereas low/absent immunoreactivity for HSF1 was detected in corresponding surrounding nontumorous livers. Subsequently, the effect of Hsf1 inactivation was investigated in the TAZS89A/ $\Delta \mathrm{N} 90-\beta$-catenin mouse model. Specifically, to achieve this goal, TAZS89A and $\Delta \mathrm{N} 90-\beta$-catenin plasmids were coinjected together with a dominant negative form of HSF1 (dnHSF1) in the mouse liver via hydrodynamic gene delivery (these mice will be referred to as TAZS89A/ $\Delta$ N90- $\beta$-catenin/dnHSF1) (Figure 7A). This approach has been found to be highly effective in completely suppressing liver carcinogenesis driven by either Akt or the c-myc protooncogene in mice. ${ }^{38,39}$ Unexpectedly, Hsf1 inactivation neither affected HB development (Figure 7B) nor changed the histopathologic features of TAZS89A/ $\Delta$ N90- $\beta$-catenin mice (Figure $7 \mathrm{C}$ ). At the microscopic level, in fact, both epithelial and mesenchymal features were retained by $\mathrm{HB}$ lesions developed in TAZS89A/ $\Delta \mathrm{N} 90-\beta$-catenin/dnHSF1 mice (Figure 7C). Furthermore, no significant differences in liver/body weight ratio between TAZS89A/ $\Delta$ N90- $\beta$-catenin and TAZS89A/DN90- $\beta$-catenin/dnHSF1 mice were detected (Figure 7D).

Taken together, the present data indicate that TAZS89A/ $\Delta$ N90- $\beta$-catenin HB development and progression do not primarily depend on Hsf1 in mice.

\section{Effects of Loss of Endogenous YAP on TAZS89A/ $\Delta N 90-\beta$-Catenin-Driven HB Formation}

Next, this study investigated whether activated YAP and TAZ have redundant and/or distinct roles in HB formation. First, the levels of Yap, the Taz paralog, were evaluated in the HB lesions from TAZS89A/ $\Delta N 90-\beta$-catenin mice. While total protein levels of Yap in TAZS89A/ $\Delta$ N90- $\beta$ catenin lesions were similar to those in wild-type livers (Supplemental Figure S1), nuclear accumulation of Yap (a sign of its activation) was detected in TAZS89A/ $\Delta$ N90- $\beta$ catenin HB lesions (Figure 8). Subsequently, we 
A

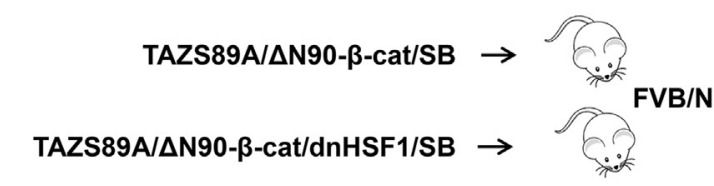

C

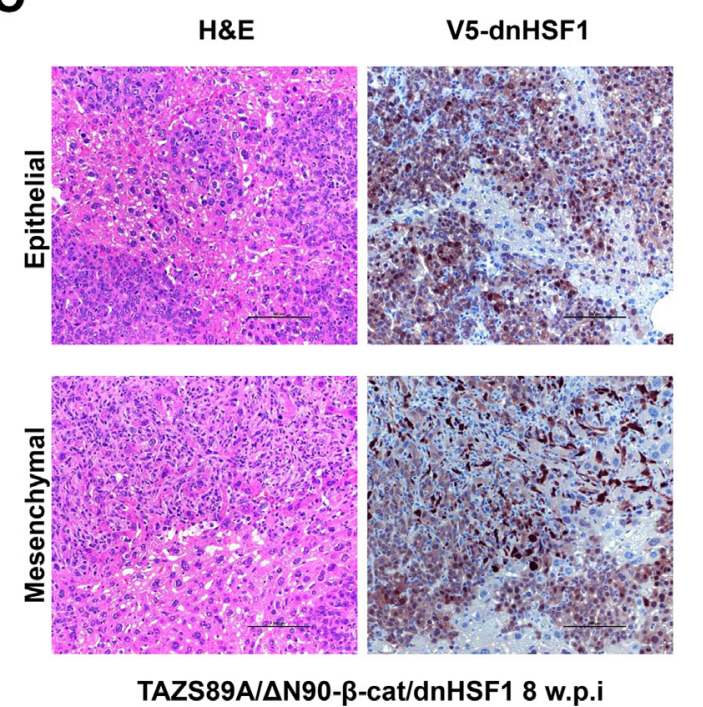

B

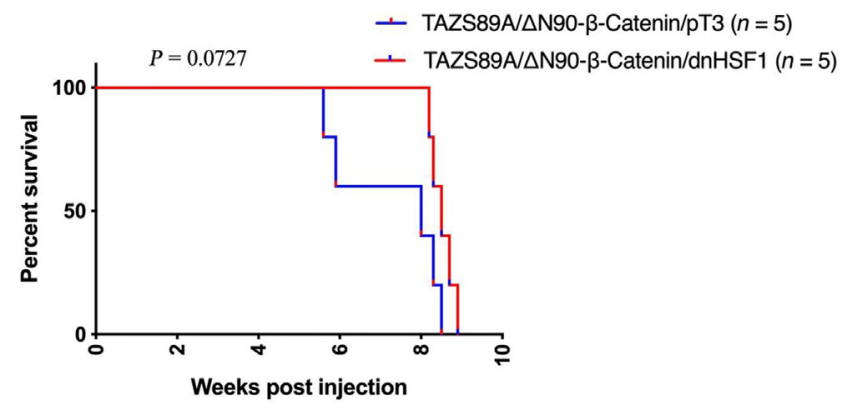

D

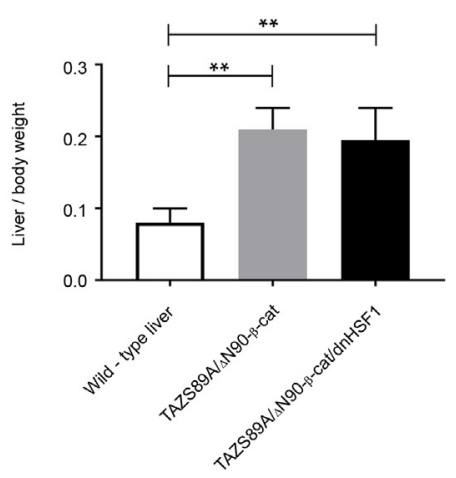

Figure 7 Inactivation of heat shock factor 1 (HSF1) does not impair liver carcinogenesis in TAZS89A/ $\Delta N 90-\beta$-catenin mice. A: Study design. B: Survival curve of TAZS89A/ $\triangle N$ N90 $-\beta$-catenin and TAZS89A/ $\Delta N 90-\beta$-catenin/dominant negative (dn)-HSF1 mice. The dnHSF1 construct is a dominant negative version of HSF1 that suppresses HSF1 activity. C: Absence of any effect on tumor development in TAZS89A/ $\Delta$ N90- $\beta$-catenin/dnHSF1 mice. Tumors from TAZS89A/ $\Delta$ N90- $\beta$ catenin/dnHSF1 mice display the presence of the epithelial and mesenchymal component. Homogeneous immunohistochemical staining for the V5-tagged dnHSF1 construct throughout the lesions indicates the effective transfection of dnHSF1 in the tumor cells. D: Liver weight/body weight ratio of wild-type as well as TAZS89A $/ \Delta$ N90- $\beta$-catenin and TAZS89A $/ \Delta N 90-\beta$-catenin/dnHSF1 mice. Data are expressed as means \pm SD (D). ${ }^{* *} P<0.01$ versus wild-type liver. Scale bars $=$ $50 \mu \mathrm{m}($ C). Original magnification, $\times 400$. H\&E, hematoxylin and eosin staining; SB, sleeping beauty transposase; w.p.i., weeks post-injection.

investigated whether TAZS89A/ $\triangle \mathrm{N} 90-\beta$-catenin overexpression induces HB development in mice in the absence of endogenous Yap protein. For this purpose, conditional Yap knockout $\left(\right.$ Yap $\left.^{\text {flox/flox }}\right)$ mice were used. ${ }^{15}$ Specifically, TAZS89A/ $\Delta$ N90 $-\beta$-catenin plasmids were hydrodynamically injected together with pCMV-Cre plasmids into $\mathrm{Yap}^{\text {flox/flox }}$ mice (TAZS89A/ $\Delta \mathrm{N} 90-\beta$-catenin/Cre). As control, TAZS89A/ $\Delta$ N90- $\beta$-catenin plasmids were mixed with pCMV empty vector and co-injected into $\mathrm{Yap}^{\text {flox/flox }}$ mice (TAZS89A/DN90- $\beta$-catenin/pCMV) (Figure 8A). Both cohorts of mice developed liver tumors and were required to be euthanized by 10 to 12 weeks after injection, without a significant difference between the two mouse cohorts in terms of survival duration (Figure 8, B, D, and E). Histologically, HB lesions from mice injected with TAZS89A/ $\triangle$ N90- $\beta$-catenin/pCMV and TAZS89A/ $\Delta \mathrm{N} 90-\beta$-catenin/Cre were indistinguishable and consisted of both the epithelial and the mesenchymal components (Figure 8D). Importantly, while cytoplasmic and nuclear staining of Yap could be detected in TAZS89A/DN90- $\beta$ catenin/pCMV HB cells, no Yap expression was found in TAZS89A/ $\Delta$ N90- $\beta$-catenin/Cre HB cells. Instead, positive cytoplasmic Yap staining was readily observed in the surrounding normal liver tissues as well as stromal cells within the HB lesions (Figure 8D). Loss of Yap expression in TAZS89A $/ \Delta N$ N0 $-\beta$-catenin/Cre tumor tissues was further validated by Western blot analysis (Figure $8 \mathrm{C}$ ).

Overall, the present data indicate that activated TAZ is able to induce HB formation in the absence of the endogenous YAP.

\section{Cooperation of TAZ with $\beta$-Catenin and YAP in the Growth of Human HB Cells in Vitro}

The findings in human and mouse HB specimens support a major role of TAZ in HB formation. To further investigate the relevance of TAZ activation in this tumor type, we examined whether TAZ expression is required for human 
A

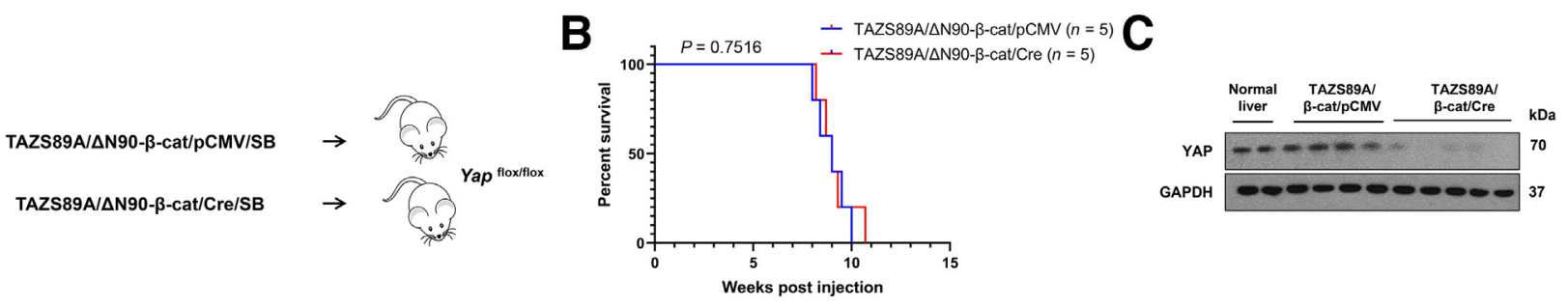

D

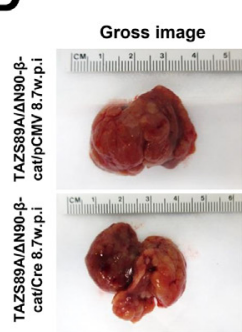

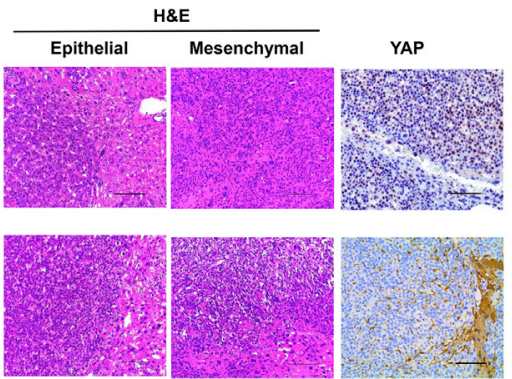

$\mathbf{E}$

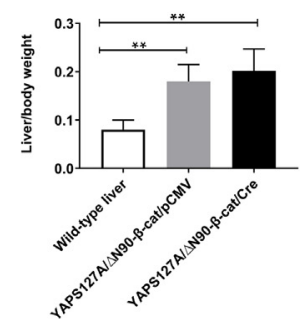

Figure 8 Deletion of yes-associated protein (Yap) does not affect liver carcinogenesis in TAZS89A/ $\Delta$ N90- $\beta$-catenin mice. A: Study design. Briefly, Yap flox/ flox conditional knockout mice were subjected to hydrodynamic tail vein injection of either TAZS89A/ $\Delta$ N90- $\beta$-catenin/pCMV (control) or TAZS89A/ $\Delta$ N90- $\beta$ catenin/Cre plasmid. B: Survival curve of TAZS89A/ $\Delta$ N90- $\beta$-catenin/pCMV and TAZS89A/ $\Delta$ N90- $\beta$-catenin/Cre mice. C: Western blot analysis showing the effective Cre-mediated deletion of Yap in the liver of TAZS89A/DN90- $\beta$-catenin/Cre mice. D: Liver lesions from TAZS89A/ $\Delta N 90-\beta$-catenin/pCMV and TAZS89A/ $\triangle$ N90- $\beta$-catenin/Cre mice are morphologically undistinguishable, both consisting of an epithelial and a mesenchymal component. As expected, TAZS89A/ $\triangle$ N90- $\beta$-catenin/pCMV livers display nuclear accumulation of Yap in the lesions (top row), which is instead lost in corresponding lesions from TAZS89A/ $\Delta \mathrm{N} 90-\beta$-catenin/Cre mice. In the latter mice, Yap cytoplasmic immunoreactivity is preserved in the non-neoplastic liver surrounding the tumors (bottom row). E: Liver weight/body weight ratio of wild-type as well as TAZS89A/ $\Delta$ N90- $\beta$-catenin/pCMV and TAZS89A/ $\Delta$ N90- $\beta$-catenin/Cre mice. Data are expressed as means \pm SD (E). ${ }^{*} P<0.01$ versus wild-type liver. Scale bars $=100 \mu \mathrm{m}$. Original magnification, $\times 200$. GAPDH, glyceraldehyde phosphate dehydrogenase; $\mathrm{H} \& \mathrm{E}$, hematoxylin and eosin staining; SB, sleeping beauty transposase.

HB cell growth in vitro. Thus, TAZ was silenced in two human HB cell lines (HepG2 and Hep293TT cells) using specific siRNA against TAZ (Supplemental Figures S12 and S13). Of note, TAZ knockdown resulted in a decrease in cell proliferation and induction of apoptosis in the two HB cell lines. These anti-growth effects were increased when the two cell lines were concomitantly subjected to $T A Z$ and $\beta$ catenin silencing. At the molecular level, suppression of TAZ did not affect $\beta$-catenin and YAP mRNA and protein levels, whereas $\beta$-catenin knockdown resulted in the downregulation of both TAZ and YAP.

Finally, it was assessed whether TAZ and YAP have redundant roles in human HB cells. TAZ and YAP genes were silenced using specific siRNAs, either alone or in combination, in HepG2 and Hep293TT cell lines (Supplemental Figures S14 and S15). Concomitant knockdown of TAZ and YAP resulted in a significantly more pronounced growth restraint when compared with the silencing of either TAZ or YAP alone. At the molecular level, knockdown of TAZ did not affect the levels of YAP, whereas silencing of YAP triggered up-regulation of TAZ. These findings suggest the existence of a compensatory mechanism inducing up-regulation of TAZ when YAP is suppressed in HB cells.

All together, the present data indicate that TAZ acts in concert with $\beta$-catenin and YAP to induce HB growth in vitro.

\section{Discussion}

HB is the most frequent pediatric tumor of the liver. ${ }^{1}$ The cellular and molecular mechanisms underlying HB development and progression remain poorly delineated. Recently, a body of evidence has unraveled the crucial role in this disease of the $\mathrm{Wnt} / \beta$-catenin signaling, which is aberrantly activated virtually in all cases. ${ }^{4,7,8}$ Nonetheless, the overexpression of oncogenic forms of $\beta$-catenin does not suffice for inducing HB formation in mice, implying the need for additional alterations to drive HB development. Recent studies showed that $\beta$-catenin cooperates with the YAP proto-oncogene, a downstream effector of the Hippo pathway, to induce rapid HB formation in the mouse liver. ${ }^{9}$ These data, together with the findings of simultaneous activation of $\beta$-catenin and YAP in human HB specimens, ${ }^{9}$ strongly support the functional importance of the Hippo pathway in this disease. In the present study, we further investigated the role of the Hippo signaling in this tumor type by focusing on the YAP ortholog, TAZ. First, the frequent activation of TAZ in human HB specimens was observed, suggesting an important role of TAZ in this tumor type. Furthermore, the activation of Taz synergizes with oncogenic $\beta$-catenin to induce HB formation in mice, thus recapitulating the effects of combining Yap and $\beta$ catenin overexpression in the mouse liver. Nonetheless, some important differences occur between TAZS89A/ $\Delta$ N90- $\beta$ catenin and YAPS127A/ $\Delta$ N90- $\beta$-catenin HB lesions. In 
particular, histopathologic analysis revealed that HB lesions from TAZS89A/ $\Delta$ N90- $\beta$-catenin display the presence of a mesenchymal component, which is instead absent in corresponding lesions from YAPS127A/ $\Delta$ N90- $\beta$-catenin mice. Although the molecular reason(s) for this histopathologic difference between the two HB models is not clear, these data unveiled the pivotal role played by the Notch pathway in this phenomenon. Indeed, suppression of the canonical Notch cascade using the dnRBP-J construct was accompanied by the disappearance of the mesenchymal phenotype in TAZS89A/ $\Delta \mathrm{N} 90-\beta$-catenin HB lesions. Additional studies are needed to better define the molecular mechanisms and the specific Notch targets involved in this event.

Another gene specifically up-regulated in TAZS89A/ $\Delta$ N90- $\beta$-catenin tumors was the HSF1 transcription factor, whose oncogenic role in liver cancer is well established. ${ }^{15,32,38}$ Unexpectedly, inactivation of Hsf1 in TAZS89A/ $\Delta$ N90- $\beta$-catenin mice had no effect on HB development. Previous studies showed that blockade of Hsf1 activity using the same strategy is able to completely suppress hepatocarcinogenesis driven by either Akt or c-myc overexpression in mice. ${ }^{15,38}$ These contrasting data suggest that the addiction to HSF1 is presumably oncogene- and context-dependent in cancer. Similarly, loss-of-function experiments using $\mathrm{Yap}^{\text {flox/flox }}$ conditional knockout mice showed that the inhibition of Yap neither suppresses nor delays HB development in TAZS89A/ $\Delta \mathrm{N} 90-\beta$-catenin mice. These in vivo findings, together with in vitro data showing an additive anti-growth effect of simultaneous silencing of TAZ and YAP in human HepG2 and Hep293TT HB cell lines, indicate that the functions of TAZ and YAP might overlap only partly in this tumor entity, at least in mice. In addition, preliminary results from our group indicate that concomitant overexpression of YAPS127A and TAZS89A in the mouse liver does not suffice for driving tumor development (X.C., unpublished data). The latter observation suggests that YAP and TAZ are not able to trigger liver tumor initiation alone or in combination, at least by the hydrodynamic gene-delivery method. However, these findings do not exclude a collaborative (and nonredundant) role of the two proto-oncogenes in driving tumor progression. Further studies using Taz and Yap conditional knockout mice should be conducted to address this important issue.

Our present data might also have potentially important therapeutic implications in this aggressive disease in humans. Indeed, the findings from mouse models and cell lines suggest that concomitant targeting of the $\mathrm{Wnt} / \beta$-catenin and Hippo pathways using specific inhibitors might be highly detrimental for HB growth. Unfortunately, these two signaling cascades are not easily druggable and no effective drugs against them are commercially available. A possible strategy would be the use of tankyrase inhibitors, which are able to suppress both oncogenic pathways and recently provided encouraging results in the treatment of hepatocellular carcinoma in experimental models. ${ }^{40,41}$

\section{Conclusion}

In summary, our findings for the first time reveal a crucial oncogenic function of TAZ in the development of $\mathrm{HB}$ as well as its collaborative role with the $\beta$-catenin signaling pathway in HB. Further studies using the TAZS89A/ $\Delta$ N90- $\beta$-catenin mouse model would be useful for elucidating the mechanisms of such cooperation and might provide a deeper understanding of the molecular pathogenesis of human $\mathrm{HB}$ as well as being helpful for its effective treatment.

\section{Acknowledgments}

We thank Ingrid Winkel and Manfred Meyer (Institute of Pathology, University of Regensburg, Regensburg, Germany) for their excellent technical work; Dr. Gail Tomlinson (University of Texas Southwestern Medical Center) for providing us with the Hep293TT cells; and Dr. Eric Olson (University of Texas Southwestern Medical Center) for kindly providing Yap $^{\text {flox/flox }}$ mice.

\section{Authors Contributions}

S.Z., J.Z., Y.Z., J.T., X.L., P.L., M.S., and Z.S. provided technical and material support; F.X., S.Z., and J.Z. analyzed and interpreted the data; K.E. and M.E. provided pathology support; A.K. and C.A. provided technical support; Z.S. contributed intellectual content; M.E. and J.T. revised the manuscript; N.C. designed the study; X.C. and D.F.C. designed the study, and wrote and revised the manuscript; A.C. designed the study, provided technical support, and revised the manuscript; all of the authors read and approved the final manuscript.

\section{Supplemental Data}

Supplemental material for this article can be found at https://doi.org/10.1016/j.ajpath.2020.03.011.

\section{References}

1. Czauderna P, Haeberle B, Hiyama E, Rangaswami A, Krailo M, Maibach R, Rinaldi E, Feng Y, Aronson D, Malogolowkin M, Yoshimura K, Leuschner I, Lopez-Terrada D, Hishiki T, Perilongo G, von Schweinitz D, Schmid I, Watanabe K, Derosa M, Meyers R: The Children's Hepatic Tumors International Collaboration (CHIC): novel global rare tumor database yields new prognostic factors in hepatoblastoma and becomes a research model. Eur J Cancer 2016, 52: 92-101

2. Meyers RL, Maibach R, Hiyama E, Haberle B, Krailo M, Rangaswami A, Aronson DC, Malogolowkin MH, Perilongo G, von Schweinitz D, Ansari M, Lopez-Terrada D, Tanaka Y, Alaggio R, Leuschner I, Hishiki T, Schmid I, Watanabe K, Yoshimura K, Feng Y, Rinaldi E, Saraceno D, Derosa M, Czauderna P: Riskstratified staging in paediatric hepatoblastoma: a unified analysis from 
the Children's Hepatic Tumors International Collaboration. Lancet Oncol 2017, 18:122-131

3. Lim IIP, Bondoc AJ, Geller JI, Tiao GM: Hepatoblastoma-the evolution of biology, surgery, and transplantation. Children (Basel) 2019, 6:1

4. Bell D, Ranganathan S, Tao J, Monga SP: Novel advances in understanding of molecular pathogenesis of hepatoblastoma: a Wnt/beta-catenin perspective. Gene Expr 2017, 17:141-154

5. Czauderna P, Garnier H: Hepatoblastoma: current understanding, recent advances, and controversies. F1000Res 2018, 7:53

6. Hooks KB, Audoux J, Fazli H, Lesjean S, Ernault T, Dugot-Senant N, Leste-Lasserre T, Hagedorn M, Rousseau B, Danet C, Branchereau S, Brugieres L, Taque S, Guettier C, Fabre M, Rullier A, Buendia MA, Commes T, Grosset CF, Raymond AA: New insights into diagnosis and therapeutic options for proliferative hepatoblastoma. Hepatology 2018, 68:89-102

7. Adebayo Michael AO, Ko S, Tao J, Moghe A, Yang H, Xu M, Russell JO, Pradhan-Sundd T, Liu S, Singh S, Poddar M, Monga JS, Liu P, Oertel M, Ranganathan S, Singhi A, Rebouissou S, ZucmanRossi J, Ribback S, Calvisi D, Qvartskhava N, Gorg B, Haussinger D, Chen X, Monga SP: Inhibiting glutamine-dependent mTORC1 activation ameliorates liver cancers driven by beta-catenin mutations. Cell Metab 2019, 29:1135-11350.e6

8. Yang A, Sisson R, Gupta A, Tiao G, Geller JI: Germline APC mutations in hepatoblastoma. Pediatr Blood Cancer 2018, 65: e26892

9. Tao J, Calvisi DF, Ranganathan S, Cigliano A, Zhou L, Singh S, Jiang L, Fan B, Terracciano L, Armeanu-Ebinger S, Ribback S, Dombrowski F, Evert M, Chen X, Monga SPS: Activation of betacatenin and Yap1 in human hepatoblastoma and induction of hepatocarcinogenesis in mice. Gastroenterology 2014, 147:690-701

10. Patel SH, Camargo FD, Yimlamai D: Hippo signaling in the liver regulates organ size, cell fate, and carcinogenesis. Gastroenterology 2017, 152:533-545

11. Fu V, Plouffe SW, Guan KL: The Hippo pathway in organ development, homeostasis, and regeneration. Curr Opin Cell Biol 2017, 49: 99-107

12. Min Q, Molina L, Li J, Adebayo Michael AO, Russell JO, Preziosi ME, Singh S, Poddar M, Matz-Soja M, Ranganathan S, Bell AW, Gebhardt R, Gaunitz F, Yu J, Tao J, Monga SP: Betacatenin and yes-associated protein 1 cooperate in hepatoblastoma pathogenesis. Am J Pathol 2019, 189:1091-1104

13. Zhang J, Liu P, Tao J, Wang P, Zhang Y, Song X, Che L, Sumazin P, Ribback S, Kiss A, Schaff Z, Cigliano A, Dombrowski F, Cossu C, Pascale RM, Calvisi DF, Monga SP, Chen X: TEA domain transcription factor 4 is the major mediator of yes-associated protein oncogenic activity in mouse and human hepatoblastoma. Am J Pathol 2019, 189:1077-1090

14. Callus BA, Finch-Edmondson ML, Fletcher S, Wilton SD: YAPping about and not forgetting TAZ. FEBS Lett 2019, 593:253-276

15. Xin M, Kim Y, Sutherland LB, Qi X, McAnally J, Schwartz RJ, Richardson JA, Bassel-Duby R, Olson EN: Regulation of insulin-like growth factor signaling by Yap governs cardiomyocyte proliferation and embryonic heart size. Sci Signal 2011, 4:ra70

16. Xu M, Wang J, Xu Z, Li R, Wang P, Shang R, Cigliano A, Ribback S, Solinas A, Pes GM, Evert K, Wang H, Song X, Zhang S, Che L, Pascale RM, Calvisi DF, Liu Q, Chen X: SNAI1 promotes the cholangiocellular phenotype, but not epithelial-mesenchymal transition, in a murine hepatocellular carcinoma model. Cancer Res 2019, 79:5563-5574

17. Zhang N, Bai H, David KK, Dong J, Zheng Y, Cai J, Giovannini M, Liu P, Anders RA, Pan D: The Merlin/NF2 tumor suppressor functions through the YAP oncoprotein to regulate tissue homeostasis in mammals. Dev Cell 2010, 19:27-38

18. Chen X, Calvisi DF: Hydrodynamic transfection for generation of novel mouse models for liver cancer research. Am J Pathol 2014, 184 : 912-923
19. Bonamassa B, Hai L, Liu D: Hydrodynamic gene delivery and its applications in pharmaceutical research. Pharm Res 2011, 28: 694-701

20. Turusov VS, Diwan BA, Engelhardt NV, Rice JM: Hepatoblastoma, mouse. Edited by Jones TC, Popp JA, Mohr U. In Digestive System. Berlin, Germany: Springer Berlin Heidelberg, 1997. pp. 91-101

21. Calvisi DF, Ladu S, Conner EA, Factor VM, Thorgeirsson SS: Dysregulation of E-cadherin in transgenic mouse models of liver cancer. Lab Invest 2004, 84:1137-1147

22. Yimlamai D, Christodoulou C, Galli GG, Yanger K, PepeMooney B, Gurung B, Shrestha K, Cahan P, Stanger BZ, Camargo FD: Hippo pathway activity influences liver cell fate. Cell 2014, 157:1324-1338

23. Lei QY, Zhang H, Zhao B, Zha ZY, Bai F, Pei XH, Zhao S, Xiong Y, Guan KL: TAZ promotes cell proliferation and epithelialmesenchymal transition and is inhibited by the hippo pathway. Mol Cell Biol 2008, 28:2426-2436

24. Stauffer JK, Scarzello AJ, Andersen JB, De Kluyver RL, Back TC, Weiss JM, Thorgeirsson SS, Wiltrout RH: Coactivation of AKT and beta-catenin in mice rapidly induces formation of lipogenic liver tumors. Cancer Res 2011, 71:2718-2727

25. Tward AD, Jones KD, Yant S, Cheung ST, Fan ST, Chen X, Kay MA, Wang R, Bishop JM: Distinct pathways of genomic progression to benign and malignant tumors of the liver. Proc Natl Acad Sci U S A 2007, 104:14771-14776

26. Lee SA, Ho C, Roy R, Kosinski C, Patil MA, Tward AD, Fridlyand J, Chen $\mathrm{X}$ : Integration of genomic analysis and in vivo transfection to identify sprouty 2 as a candidate tumor suppressor in liver cancer Hepatology 2008, 47:1200-1210

27. Soini T, Haveri H, Elo JM, Kauppinen M, Kyronlahti A, Salo MK, Lohi J, Andersson LC, Wilson DB, Heikinheimo M: Transcription factor GATA-4 is abundantly expressed in childhood but not in adult liver tumors. J Pediatr Gastroenterol Nutr 2012, 54:101-108

28. Sumazin P, Chen Y, Trevino LR, Sarabia SF, Hampton OA, Patel K, Mistretta TA, Zorman B, Thompson P, Heczey A, Comerford S, Wheeler DA, Chintagumpala M, Meyers R, Rakheja D, Finegold MJ, Tomlinson G, Parsons DW, Lopez-Terrada D: Genomic analysis of hepatoblastoma identifies distinct molecular and prognostic subgroups. Hepatology 2017, 65:104-121

29. Zhang H, Liu CY, Zha ZY, Zhao B, Yao J, Zhao S, Xiong Y, Lei QY, Guan KL: TEAD transcription factors mediate the function of TAZ in cell growth and epithelial-mesenchymal transition. J Biol Chem 2009, 284:13355-13362

30. Raghunath A, Sundarraj K, Arfuso F, Sethi G, Perumal E: Dysregulation of Nrf2 in hepatocellular carcinoma: role in cancer progression and chemoresistance. Cancers (Basel) 2018, 10:481

31. Menegon S, Columbano A, Giordano S: The dual roles of NRF2 in cancer. Trends Mol Med 2016, 22:578-593

32. Jin X, Moskophidis D, Mivechi NF: Heat shock transcription factor 1 is a key determinant of HCC development by regulating hepatic steatosis and metabolic syndrome. Cell Metab 2011, 14 91-103

33. Tschaharganeh DF, Chen X, Latzko P, Malz M, Gaida MM, Felix K, Ladu S, Singer S, Pinna F, Gretz N, Sticht C, Tomasi ML, Delogu S, Evert M, Fan B, Ribback S, Jiang L, Brozzetti S, Bergmann F, Dombrowski F, Schirmacher P, Calvisi DF, Breuhahn K: Yes-associated protein up-regulates jagged-1 and activates the Notch pathway in human hepatocellular carcinoma. Gastroenterology 2013, 144: $1530-1542 . e 12$

34. Wang J, Dong M, Xu Z, Song X, Zhang S, Qiao Y, Che L, Gordan J, Hu K, Liu Y, Calvisi DF, Chen X: Notch2 controls hepatocytederived cholangiocarcinoma formation in mice. Oncogene 2018, 37: 3229-3242

35. Zhang S, Wang J, Wang H, Fan L, Fan B, Zeng B, Tao J, Li X, Che L, Cigliano A, Ribback S, Dombrowski F, Chen B, Cong W, Wei L, Calvisi DF, Chen X: Hippo cascade controls lineage 
commitment of liver tumors in mice and humans. Am J Pathol 2018, 188:995-1006

36. Litten JB, Chen TT, Schultz R, Herman K, Comstock J, Schiffman J, Tomlinson GE, Rakheja D: Activated NOTCH2 is overexpressed in hepatoblastomas: an immunohistochemical study. Pediatr Dev Pathol 2011, 14:378-383

37. Che L, Fan B, Pilo MG, Xu Z, Liu Y, Cigliano A, Cossu A, Palmieri G, Pascale RM, Porcu A, Vidili G, Serra M, Dombrowski F, Ribback S, Calvisi DF, Chen X: Jagged 1 is a major Notch ligand along cholangiocarcinoma development in mice and humans. Oncogenesis 2016, 5:e274

38. Cigliano A, Pilo MG, Li L, Latte G, Szydlowska M, Simile MM, Paliogiannis P, Che L, Pes GM, Palmieri G, Sini MC, Cossu A, Porcu A, Vidili G, Seddaiu MA, Pascale RM, Ribback S, Dombrowski F, Chen X, Calvisi DF: Deregulated c-Myc requires a functional HSF1 for experimental and human hepatocarcinogenesis. Oncotarget 2017, 8:90638-90650

39. Cigliano A, Wang C, Pilo MG, Szydlowska M, Brozzetti S, Latte G, Pes GM, Pascale RM, Seddaiu MA, Vidili G, Ribback S, Dombrowski F, Evert M, Chen X, Calvisi DF: Inhibition of HSF1 suppresses the growth of hepatocarcinoma cell lines in vitro and AKT-driven hepatocarcinogenesis in mice. Oncotarget 2017, 8: 54149-54159

40. Ma L, Wang X, Jia T, Wei W, Chua MS, So S: Tankyrase inhibitors attenuate WNT/beta-catenin signaling and inhibit growth of hepatocellular carcinoma cells. Oncotarget 2015, 6:25390-25401

41. Jia J, Qiao Y, Pilo MG, Cigliano A, Liu X, Shao Z, Calvisi DF, Chen X: Tankyrase inhibitors suppress hepatocellular carcinoma cell growth via modulating the Hippo cascade. PLoS One 2017, 12: e0184068 